\title{
Ramsey precompact expansions of homogeneous directed graphs
}

\author{
Jakub Jasiński \\ University of Calgary \\ Department of Mathematics and Statistics \\ Calgary, Alberta, Canada T2N 1N4 \\ jjasinsk@ucalgary.ca \\ Lionel Nguyen Van Thé ${ }^{\dagger}$ \\ Aix-Marseille Université \\ CNRS, Centrale Marseille \\ I2M, UMR 7373 \\ 13453 Marseille, France \\ lionel@latp.univ-mrs.fr
}

\author{
Claude Laflamme* \\ University of Calgary \\ Department of Mathematics and Statistics \\ Calgary, Alberta, Canada T2N 1N4 \\ laflamme@ucalgary.ca \\ Robert Woodrow \\ University of Calgary \\ Department of Mathematics and Statistics \\ Calgary, Alberta, Canada T2N 1N4 \\ woodrow@ucalgary.ca
}

Submitted: Oct 24, 2013; Accepted: Nov 18, 2014; Published: Nov 27, 2014

Mathematics Subject Classifications: 05D10; 03C13; 03C15; 05C20; 37B05; 54H20

\begin{abstract}
In 2005, Kechris, Pestov and Todorčević provided a powerful tool to compute an invariant of topological groups known as the universal minimal flow, immediately leading to an explicit representation of this invariant in many concrete cases. More recently, the framework was generalized allowing for further applications, and the purpose of this paper is to apply these new methods in the context of homogeneous directed graphs.

In this paper, we show that the age of any homogeneous directed graph allows a Ramsey precompact expansion. Moreover, we verify the relative expansion properties and consequently describe the respective universal minimal flows.
\end{abstract}

\section{Introduction}

The article [KPT05] by Kechris, Pestov and Todorčević established a fundamental correspondence between structural Ramsey theory and topological dynamics. As an immediate

*Supported by NSERC of Canada Grant \# 690404

${ }^{\dagger}$ Supported by A.N.R. project Grupoloco (ANR-11-JS01-0008) 
consequence, it triggered a renewed interest for structural Ramsey theory, and also motivated a more detailed investigation of the connection between combinatorics and Polish group actions. More precisely, [KPT05] provided an extremely powerful tool to compute an invariant known as the universal minimal flow, and immediately led to an explicit representation of this invariant in many concrete cases, including the case of most homogeneous (simple, loopless) graphs. However, in some particular situations including some homogeneous directed graphs, that framework did not allow to perform the computation directly.

Recently, the ordering property technique was generalized in [NVT13] to the notion of precompact expansion, opening the door for further applications. This was in particular used to compute the universal minimal flows of the automorphism groups of the homogeneous circular directed graphs $\mathbf{S}(2)$ and $\mathbf{S}(3)$. The purpose of this paper is to apply these new methods in the context of homogeneous directed graphs; following the classification by Cherlin in [C98], we show that in each case its age allows a Ramsey precompact expansion. In this paper, it will be shown that in addition, this expansion can be used to compute the universal minimal flow of the corresponding automorphism group.

The underlying strategy we will use in order to construct Ramsey expansions can be described as follows: starting from a homogeneous directed graph, if the new age is not Ramsey by simply adding any well-chosen linear ordering, then further expand using finitely many additional relations.

In an effort to make the current paper relatively self-contained, we review the main results and notation of [KPT05] and [NVT13] relevant to the current work. In what follows, $\mathbb{N}$ denotes the set $\{0,1,2, \ldots\}$ of natural numbers, and for a natural number $m$, $[m]$ will denote the set $\{0, \ldots, m-1\}$. We will assume that the reader is familiar with the concepts of first order logic, first order structures, Fraïssé theory (cf [KPT05], section 2), reducts and expansions (cf [KPT05], section 5). If $L$ is a first order signature and $\mathbf{A}$ and $\mathbf{B}$ are $L$-structures, we will write $\mathbf{A} \leqslant \mathbf{B}$ when $\mathbf{A}$ embeds in $\mathbf{B}, \mathbf{A} \subset \mathbf{B}$ when $\mathbf{A}$ is a substructure of $\mathbf{B}$, and $\mathbf{A} \cong \mathbf{B}$ when $\mathbf{A}$ and $\mathbf{B}$ are isomorphic. If $C$ is a subset of the universe of $\mathbf{A}$ which supports a substructure of $\mathbf{A}$, we will write $\mathbf{A} \uparrow C$ for the corresponding substructure.

A Fraïssé class in a countable first order relational language $L$ will be a countable class of finite $L$-structures of arbitrarily large finite sizes, satisfying the hereditarity, joint embedding and amalgamation property, and a Fraïssé structure (or Fraïssé limit) in $L$ will be a countable, locally finite, homogeneous $L$-structure. In general a relational structure is called homogeneous if any isomorphism between finite substructures can be extended to an automorphism of the entire structure. In [KPT05], two combinatorial properties of classes of finite structures have a considerable importance, those are the Ramsey property and the ordering property. In order to define the Ramsey property, let $k, l \in \mathbb{N}$, and $\mathbf{A}, \mathbf{B}, \mathbf{C}$ be $L$-structures. The set of all copies of $\mathbf{A}$ in $\mathbf{B}$ is written using the binomial notation

$$
\left(\begin{array}{l}
\mathbf{B} \\
\mathbf{A}
\end{array}\right)=\left\{\mathbf{A}^{\prime} \subset \mathbf{B}: \mathbf{A}^{\prime} \cong \mathbf{A}\right\}
$$


We use the standard arrow partition symbol

$$
\mathbf{C} \longrightarrow(\mathbf{B})_{k, l}^{\mathbf{A}}
$$

to mean that for every map $c:\left(\begin{array}{l}\mathbf{C} \\ \mathbf{A}\end{array}\right) \longrightarrow[k]$, thought as a $k$-coloring of the copies of $\mathbf{A}$ in $\mathbf{C}$, there is a copy $\mathbf{B}^{\prime} \in\left(\begin{array}{l}\mathbf{C} \\ \mathbf{B}\end{array}\right)$ such that $c$ takes at most $l$-many values on $\left(\begin{array}{c}\mathbf{B}^{\prime} \\ \mathbf{A}\end{array}\right)$. When $l=1$, this is written

$$
\mathbf{C} \longrightarrow(\mathbf{B})_{k}^{\mathbf{A}}
$$

A class $\mathcal{K}$ of finite $L$-structures is then said to have the Ramsey property when the following statement holds:

$$
\forall k \in \mathbb{N} \quad \forall \mathbf{A}, \mathbf{B} \in \mathcal{K} \quad \exists \mathbf{C} \in \mathcal{K} \quad \mathbf{C} \longrightarrow(\mathbf{B})_{k}^{\mathbf{A}} .
$$

When $\mathcal{K}=\operatorname{Age}(\mathbf{F})$, where $\mathbf{F}$ is a Fraïssé structure, this is equivalent, via a compactness argument to:

$$
\forall k \in \mathbb{N} \quad \forall \mathbf{A}, \mathbf{B} \in \mathcal{K} \quad \mathbf{F} \longrightarrow(\mathbf{B})_{k}^{\mathbf{A}} .
$$

As for the ordering property used in [KPT05], assume that $<$ is a binary relation symbol not contained in $L$, and that $L^{*}=L \cup\{<\}$. For $\mathcal{K}$, a Fraïssé class in $L$, we write $\mathcal{K}^{*}$ for an order expansion of $\mathcal{K}$ in $L^{*}$. This means that all elements of $\mathcal{K}^{*}$ are of the form $\mathbf{A}^{*}=\left(\mathbf{A},<^{\mathbf{A}}\right)$, where $\mathbf{A} \in \mathcal{K}$ and $<^{\mathbf{A}}$ is a linear ordering on the universe $A$ of $\mathbf{A}$; thus $\mathbf{A}$ is then the reduct of $\mathbf{A}^{*}$ to $L$ and is also denoted $\mathbf{A}^{*} \uparrow L$, or even $\mathbf{A}^{*} \uparrow \mathcal{K}$, and that, conversely, any $\mathbf{A} \in \mathcal{K}$ admits a linear ordering $<^{\mathbf{A}}$ so that $\left(\mathbf{A},<^{\mathbf{A}}\right) \in \operatorname{Age}\left(\mathcal{K}^{*}\right)$. Then, $\mathcal{K}^{*}$ is said to satisfy the ordering property relative to $\mathcal{K}$ if, for every $\mathbf{A} \in \mathcal{K}$, there exists $\mathbf{B} \in \mathcal{K}$ such that

$$
\forall \mathbf{A}^{*}, \mathbf{B}^{*} \in \mathcal{K}^{*} \quad\left(\mathbf{A}^{*} \uparrow L=\mathbf{A} \wedge \mathbf{B}^{*} \uparrow L=\mathbf{B}\right) \Rightarrow \mathbf{A}^{*} \leqslant \mathbf{B}^{*}
$$

The ordering property is precisely the technique that was generalized in [NVT13] to precompact (relational) expansions. For such expansions, we do not require $L^{*}=L \cup\{<\}$, but only $L^{*}=L \cup\left\{R_{i}: i \in I\right\}$, where $I$ is countable, and every symbol $R_{i}$ is relational and not in $L$. An expansion $\mathbf{F}^{*}$ of $\mathbf{F}$ is then called precompact when any $\mathbf{A} \in \operatorname{Age}(\mathbf{F})$ only has finitely many expansions in $\operatorname{Age}\left(\mathbf{F}^{*}\right)$.

In the spirit as above, we say that $\mathcal{K}^{*}$ has the expansion property relative to $\mathcal{K}$ if and only if for every $\mathbf{A} \in \mathcal{K}$, there is $\mathbf{B} \in \mathcal{K}$ such that

$$
\forall \mathbf{A}^{*}, \mathbf{B}^{*} \in \mathcal{K}^{*} \quad\left(\mathbf{A}^{*} \uparrow L=\mathbf{A} \wedge \mathbf{B}^{*} \uparrow L=\mathbf{B}\right) \Rightarrow \mathbf{A}^{*} \leqslant \mathbf{B}^{*}
$$

The importance of the new technique is demonstrated in the following result, generalizing the results of [KPT05] where the ordering property had been used:

Theorem 1.1. [NVT13] Let $\boldsymbol{F}$ be a Fraïssé structure, and $\boldsymbol{F}^{*}=\left(\boldsymbol{F}, \vec{R}^{*}\right)$ a (not necessarily Fraïssé) precompact relational expansion of $\boldsymbol{F}$. Then the following are equivalent:

i) The logic action of $\operatorname{Aut}(\mathbf{F})$ on the space $\overline{\operatorname{Aut}(\mathbf{F}) \cdot \vec{R}^{*}}$ is minimal.

ii) $\operatorname{Age}\left(\boldsymbol{F}^{*}\right)$ has the expansion property relative to $\operatorname{Age}(\boldsymbol{F})$. 
Theorem 1.2. [NVT13] Let $\boldsymbol{F}$ be a Fraïssé structure, $\boldsymbol{F}^{*}=\left(\boldsymbol{F}, \vec{R}^{*}\right)$ be a Fraïssé precompact relational expansion of $\boldsymbol{F}$, and further assume that $\operatorname{Age}\left(\boldsymbol{F}^{*}\right)$ consists of rigid elements. Then the following are equivalent:

i) The logic action of $\operatorname{Aut}(\mathbf{F})$ on the space $\overrightarrow{\operatorname{Aut}(\mathbf{F}) \cdot \vec{R}^{*}}$ is its universal minimal flow.

ii) The class $\operatorname{Age}\left(\boldsymbol{F}^{*}\right)$ has the Ramsey property as well as the expansion property relative to $\operatorname{Age}(\boldsymbol{F})$.

Thus, given $\mathbf{F}$ a Fraïssé structure, our general objective is to find an expansion $\mathbf{F}^{*}$ of $\mathbf{F}$ that is precompact, has the expansion property, its age is Ramsey and consists of rigid members. Note that if the age of $F^{*}$ consists of rigid members and satisfies the Ramsey property and the joint embedding property, then it also has the amalgamation property (see [KPT05]). In this paper, we complete this program for all homogeneous directed graphs, moreover we do so using a well chosen and minimal number of new relations. All arguments are very much standard, except those used for the case of the semigeneric directed graph in Section 10.

\section{Homogeneous Directed Graphs}

A graph $G$ is a pair $(V(G), E(G))$ where $V(G)$ is the set of vertices and $E(G)$ is the set of (undirected) edges. A simple (or undirected) graph is one where $E$ is a symmetric (and irreflexive), and a directed graph is one where $E$ is asymmetric (and irreflexive). A tournament is a complete directed graph, that is every pair of distinct vertices supports exactly one directed edge. A hypergraph is a generalization of a graph where edges are non-empty subsets, and we call it a $k$-uniform hypergraph if all these edges have the same size $k$. A (2)-set system in the sense of [NR89] is a linearly ordered 2-uniform hypergraph where edges also carry a label. The following Ramsey result from [NR89] will play a central role in this paper.

Theorem 2.1. [NR89] Given two finite set systems $A$ and $\boldsymbol{B}$ and $r \in \mathbb{N}$, there is a set system $C$ such that

$$
C \rightarrow(B)_{r}^{A}
$$

For example a linearly ordered tournament can be converted into a set system as follows: if $(x, y)$ is a directed edge, then form the hyperedge $\{x, y\}$ and label it with 0 if $x<y, 1$ otherwise. Vice-versa, a set system can be converted into a directed graph by following the reverse procedure.

By identifying graphs as relational structures with universe $V(G)$ and single relation $E(G)$, it is thus natural to consider homogeneous graphs. To complete our program, we will follow the classification of homogeneous directed graphs as described in [C98]. Concretely, it means that we will construct a precompact Ramsey expansion for each of the directed graphs in Cherlin's list, which we repeat here for convenience (more detail about each object can be found in the corresponding section, or in [C98], p.74-75): 
1. $I_{n}$.

2. $C_{3}, \mathbb{Q}, \mathbf{S}(2), T^{\omega}$.

3. $\mathbf{T}\left[I_{n}\right]$ (Section 4 and 5 ), $I_{n}[\mathbf{T}]$ (Section 6 and 7 ), where $\mathbf{T}$ is a homogeneous tournament.

4. $\hat{\mathbf{T}}$ for $\mathbf{T}=I_{1}, C_{3}, \mathbb{Q}, T^{\omega}$ (Section 4$)$.

5. Complete $n$-partite (Section 8).

6. Semigeneric (Section 10).

7. $\mathbf{S}(3)$.

8. $\mathcal{P}$.

9. $\mathcal{P}(3)$ (section 9).

10. $\Gamma_{n}$.

11. $\mathcal{T}$-generic.

The cases 1, 2, 7, 8, 10, 11 will not be treated here as Ramsey precompact expansions with the expansion property are already known for them. Precisely:

1. $I_{n}$ denotes the edgeless directed graph on $n$ vertices, $n \leqslant \omega$. When $n<\omega, I_{n}$ is finite and the relevant expansion $I_{n}^{*}$ is obtained by adding $n$ unary relations, one for each point in $I_{n}$. When $n=\omega, I_{\omega}^{*}$ is obtained by adding a linear ordering that makes it isomorphic to the rationals ([KPT05], based on the usual finite Ramsey theorem).

2. These are the homogeneous tournaments. $C_{3}$ denotes the 3-cycle, and the corresponding expansion $C_{3}^{*}$ is obtained in the same way as it is obtained for $I_{n}$ by adding three unary predicates. For convenience, we will also assume that $C_{3}^{*}$ is equipped with a linear ordering $<C_{3}^{*}$. This does not change the automorphism group of $C_{3}^{*}$ but will be useful in Section $4 . \mathbb{Q}$ denotes the rationals, seen as a directed graph where $E(x, y)$ iff $x<y$. For this structure, simply take $\mathbb{Q}^{*}=\mathbb{Q}$, and the Ramsey property follows again in virtue of the usual finite Ramsey Theorem. $\mathbf{S}(2)$ denotes the dense local order; its points are the points lying at a rational angle on the unit circle, with the edge relation defined by $E(x, y)$ iff the angle from $x$ to $y$ is in the range $(0, \pi) \cdot \mathbf{S}(2)^{*}$ is constructed by adding two unary relations, corresponding to the partition into left and right half (note that there are no antipodal points at $\pi / 2$ and $3 \pi / 2$ ). Observe that there is a definable linear ordering $<^{\mathbf{S}(2)^{*}}$ in that structure, obtained by reversing edges that are between elements lying in different parts (see [NVT13], Proposition 10, for more details). In what follows, it will be convenient to include it as a relation of $\mathbf{S}(2)^{*}$. The underlying Ramsey theorem 
for this class is proved in [LNS10], and the universal minimal flow is described in [NVT13]. Finally, $T^{\omega}$ denotes the generic tournament, and $T^{\omega *}$ is obtained by adding a generic linear ordering; the corresponding Ramsey theorem is a consequence of a general result proved independently by Abramson-Harrington [AH78] and Nešetřil-Rödl [NR77, NR83, NR89].

7. $\mathbf{S}(3)$ is a variant of $\mathbf{S}(2)$, where $E(x, y)$ iff the angle from $x$ to $y$ is in the range $(0,2 \pi / 3)$. $\mathbf{S}(3)^{*}$ is constructed by adding three unary relations, corresponding to the partition into three arcs of same length and without extremity points (same references as for $\mathbf{S}(2)$ ). As for $\mathbf{S}(2)^{*}$, there is a definable linear ordering $<\mathbf{S}(3)^{*}$ in $\mathbf{S}(3)^{*}$ (see [NVT13], Proposition 12), which we will consider as a relation in $\mathbf{S}(3)^{*}$.

8. $\mathcal{P}$ denotes the generic partial order. Its expansion is obtained by considering $\mathcal{P}^{*}$, the Fraïssé limit of the class of all finite partial orders that are totally ordered by a linear extension (see [PTW84], based on unpublished results of Nešetřil-Rödl).

10. $\Gamma_{n}$ denotes the generic directed graph where $I_{n+1}$ does not embed. When $n=1$, this is nothing else than $T^{\omega}$, for which the relevant expansion was already described. When $n>1, \Gamma_{n}^{*}$ is also obtained by adding a generic linear ordering. The underlying Ramsey theorem is covered by general results of Nešetřil-Rödl thanks to the partite construction: by switching edges and non-edges, the class becomes the class of $K_{n^{-}}$ free graphs, which is treated by [NR89].

11. $\mathcal{T}$-generic, where $\mathcal{T}$ denotes a set of finite tournaments, refers to the generic directed graph as the Fraïssé limit of all the finite directed graphs which do not embed any member of $\mathcal{T}$. As in case 10, the general results by Nešetřil-Rödl allow to prove that the relevant expansion is obtained by adding a generic linear ordering.

\section{A Ramsey Lemma for Products}

This section is devoted to an elementary but powerful finite combinatorial principle.

Definition 3.1. Let $n \in \mathbb{N}^{+}$and $0 \leqslant a_{i} \leqslant b_{i} \in \mathbb{N}$ for $1 \leqslant i \leqslant n$. If $B_{i} \subseteq \mathbb{N}$ are disjoint sets of size $\left|B_{i}\right|=b_{i}$ for $1 \leqslant i \leqslant n$, then define

$$
\left(\begin{array}{c}
B_{1}, \ldots, B_{n} \\
a_{1}, \ldots, a_{n}
\end{array}\right)
$$

to consist of all sets of the form $A^{\prime}=\bigcup_{i=1}^{n} A_{i}$, where $A_{i} \subseteq B_{i}$ and $\left|A_{i}\right|=a_{i}$ for $1 \leqslant i \leqslant n$.

Using this notation, we have the following Ramsey lemma for products.

Lemma 3.2 ([GRS90]). Let $r, n \in \mathbb{N}^{+}$and let $0 \leqslant a_{i} \leqslant b_{i} \in \mathbb{N}$ for $1 \leqslant i \leqslant n$. Then there exist disjoint $C_{i} \subseteq \mathbb{N}$ for $1 \leqslant i \leqslant n$, such that given any colouring $\chi$ of

$$
\left(\begin{array}{c}
C_{1}, \ldots, C_{n} \\
a_{1}, \ldots, a_{n}
\end{array}\right)
$$


with $r$ colours, there exists $B_{i} \subseteq C_{i}$ and $\left|B_{i}\right|=b_{i}$ so that $\chi$ is monochromatic on

$$
\left(\begin{array}{c}
B_{1}, \ldots, B_{n} \\
a_{1}, \ldots, a_{n}
\end{array}\right)
$$

We symbolize the statement above with

$$
\left(C_{1}, \ldots, C_{n}\right) \rightarrow\left(b_{1}, \ldots, b_{n}\right)_{r}^{\left(a_{1}, \ldots, a_{n}\right)}
$$

If $b=b_{i}$ for all $i$, we set $N=\max \left\{\left|C_{i}\right|: 1 \leqslant i \leqslant n\right\}$ and write

$$
N \rightarrow(b)_{r}^{\left(a_{1}, \ldots, a_{n}\right)} .
$$

There is an ordered version of the above lemma. Consider the class $\mathcal{O P} \mathcal{P}_{n}$ of structures of the form $\mathbf{A}=\left(A,<^{\mathbf{A}}, P_{1}^{\mathbf{A}}, \ldots, P_{n}^{\mathbf{A}}\right)$ where $<^{\mathbf{A}}$ is a linear ordering on $A$, while $\left\{P_{i}\right\}_{1 \leqslant i \leqslant n}$ is partition of $A$ into disjoint sets. It follows from the result in [KPT05] (see proof of Theorem 8.4) that $\mathcal{O P}_{n}$ satisfies the Ramsey property. We will also have to consider sequences of elements of $\mathcal{O P}{ }_{n}$ and hence require the following Ramsey result which follows from Theorem 2 for sequences of classes in [S12].

Lemma 3.3. Let $n, k, r \in \mathbb{N}$. Let $\left(\mathbf{A}_{1}, \ldots, \mathbf{A}_{n}\right)$ and $\left(\mathbf{B}_{1}, \ldots, \mathbf{B}_{n}\right)$ be sequences of elements in $\mathcal{O} \mathcal{P}_{k}$. Then there is a sequence $\left(\mathbf{C}_{1}, \ldots, \mathbf{C}_{n}\right)$ of elements in $\mathcal{O P}_{k}$ such that for any colouring $\chi$ of sequences $\left(\mathbf{A}_{1}^{\prime}, \ldots, \mathbf{A}_{n}^{\prime}\right)$ such that $\mathbf{A}_{i}^{\prime} \in\left(\begin{array}{c}\mathbf{C}_{i} \\ \mathbf{A}_{i}\end{array}\right)$ with $r$ colours, there is a sequence $\left(\mathbf{B}_{1}^{\prime}, \ldots, \mathbf{B}_{n}^{\prime}\right)$ such that $\mathbf{B}_{i}^{\prime} \in\left(\begin{array}{l}\mathbf{C}_{i} \\ \mathbf{B}_{i}\end{array}\right)$ and $\chi$ assumes only one colour on the set of sequences $\left(\mathbf{A}_{1}^{\prime \prime}, \ldots, \mathbf{A}_{n}^{\prime \prime}\right)$ such that $\mathbf{A}_{i}^{\prime \prime} \in\left(\begin{array}{c}\mathbf{B}_{i}^{\prime} \\ \mathbf{A}_{i}\end{array}\right)$.

Furthermore, we can make the $C_{i}$ 's disjoint and consequently extend the orders to an ordering of $\bigcup_{i} C_{i}$, convex with respect to $C_{i}$ 's.

\section{$4 \mathbf{T}\left[\boldsymbol{I}_{n}\right], \hat{\mathbf{T}}$}

We are now ready to undertake our program starting with the above homogeneous directed graphs. We start with a brief description of each of them.

When $\mathbf{T}$ is a homogeneous tournament and $n$ is a positive integer, the structure $\mathbf{T}\left[I_{n}\right]$ is defined as the homogeneous directed graph whose vertex set consists of pairs $(x, i)$ with $x \in T$ and $i \in[n]$. A pair $((x, i),(y, j))$ is an edge of $\mathbf{T}\left[I_{n}\right]$ if and only if $(x, y)$ is an edge of $\mathbf{T}$.

The structure $\hat{\mathbf{T}}$ is identical to $\mathbf{T}\left[I_{2}\right]$ except that $((y, i),(x, j))$ is an edge of $\hat{\mathbf{T}}$ if and only if $(x, y)$ is an edge of $\mathbf{T}$ and $i \neq j$.

We now turn to the description of the corresponding expansions $\mathbf{T}\left[I_{n}\right]^{*}$ and $\hat{\mathbf{T}}^{*}$. To construct the structure $\mathbf{T}\left[I_{n}\right]^{*}$, we start from $\mathbf{T}^{*}$ as described at the end of Section 2 and use it to naturally expand $\mathbf{T}\left[I_{n}\right]$. Precisely, consider the language $L_{\mathbf{T}^{*}}$ of $\mathbf{T}^{*}$. It contains the edge symbol $E$ from $L_{\mathbf{T}}$ and a distinguished relation symbol $<$, interpreted as a linear ordering $<^{*}$ in $\mathbf{T}^{*}$. For $R$ is a relational symbol in $L_{\mathbf{T}^{*}} \backslash\{E,<\}$, set $R^{\mathbf{T}\left[I_{n}\right]^{*}}(\vec{u})$ iff $R^{\mathbf{T}^{*}}(\vec{x})$, where $\vec{u}=\left(\left(x_{1}, i_{1}\right), \ldots,\left(x_{n}, i_{n}\right)\right)$. As for $<$, interpret it as the lexicographical ordering 
of $<^{*}$ and the usual order on $[n]$. Finally, add to the resulting structure $n$ new unary predicates $\left(L_{i}\right)_{i \in[n]}$ defined by $L_{i}(x, j)$ iff $j=i$. The structure $\hat{\mathbf{T}}^{*}$ is constructed similarly.

It is clear that those expansions are precompact since we added only finitely many predicates. We now show that their age has the Ramsey property. In order to do this, it suffices to concentrate on the case of $\mathbf{T}\left[I_{n}\right]^{*}$, as the proof in the two other cases follows the same scheme. Let $\mathbf{A}$ be a finite substructure of $\mathbf{T}\left[I_{n}\right]^{*}$. Let $\varphi: A \rightarrow[n]$ be such that $\varphi(x)=i$ if $L_{i}(x)$. Define the collapse of $\mathbf{A}, \operatorname{coll}(\mathbf{A})$, to be the projection of $\mathbf{A}$ to $\mathbf{T}$, i.e., $x$ is in the universe of coll(A) if and only if $(x, i) \in A$ for some $i$. Construct the sequence

$$
\operatorname{recipe}(\mathbf{A})=\left(\left\{n_{1}^{1}, \ldots, n_{k_{1}}^{1}\right\}, \ldots,\left\{n_{1}^{l}, \ldots, n_{k_{l}}^{l}\right\}\right)
$$

as follows: Enumerate coll $(\mathbf{A})=x_{1}<\cdots<x_{l}$ consistently with the ordering on $\mathbf{T}^{*}$. Set $A_{x}=A \cap x \times[n]$, and let $\left\{n_{1}^{j}, \ldots, n_{k_{j}}^{j}\right\}=\varphi^{\prime \prime} A_{x_{j}}$.

Conversely, given a sequence

$$
\sigma=\left(\left\{n_{1}^{1}, \ldots, n_{k_{1}}^{1}\right\}, \ldots,\left\{n_{1}^{l}, \ldots, n_{k_{l}}^{l}\right\}\right)
$$

where $n_{j}^{i} \in[n]$ and given a finite substructure of $\mathbf{A}$ of $\mathbf{T}$ of size $l$, define $\operatorname{lift}(\mathbf{A}, \sigma)$ as follows: Enumerate $\mathbf{A}=x_{1}<\cdots<x_{l}$ consistently with the ordering on $\mathbf{T}^{*}$. The universe of $\operatorname{lift}(\mathbf{A}, \sigma)$ consists of all pairs $\left(x_{i}, n_{j}^{i}\right)$ with $1 \leqslant j \leqslant k_{i}$.

Thus we have the following immediate Lemma.

Lemma 4.1. Suppose $\mathbf{A} \cong \mathbf{A}^{\prime}$ are finite substructures of $\mathbf{T}\left[I_{n}\right]^{*}$. Then

$$
\operatorname{lift}\left(\operatorname{coll}\left(\mathbf{A}^{\prime}\right), \operatorname{recipe}(\mathbf{A})\right)=\mathbf{A}^{\prime} .
$$

Theorem 4.2. The class Age $\left(\mathbf{T}\left[I_{n}\right]^{*}\right)$ satisfies the Ramsey Property.

Proof. Let A, B be finite substructures of $\mathbf{T}\left[I_{n}\right]^{*}$ and $r \in \mathbb{N}$. We need to produce a finite substructure $\mathbf{C} \subseteq \mathbf{T}\left[I_{n}\right]^{*}$ such that

$$
\mathbf{C} \rightarrow(\mathbf{B})_{r}^{\mathbf{A}}
$$

Because Age $\left(\mathbf{T}^{*}\right)$ satisfies the Ramsey Property, there exists a finite substructure $\mathbf{C}_{0} \subseteq \mathbf{T}^{*}$ such that

$$
\mathbf{C}_{0} \rightarrow(\operatorname{coll}(\mathbf{B}))_{r}^{\operatorname{coll}(\mathbf{A})}
$$

Let $\mathbf{C}$ be the substructure of $\mathbf{T}\left[I_{n}\right]^{*}$ obtained from $\mathbf{C}_{0}$ following the same procedure that turned $\mathbf{T}^{*}$ into $\mathbf{T}\left[I_{n}\right]^{*}$. We claim that $\mathbf{C}$ is as required. Let $r \geqslant 2$ and

$$
\chi:\left(\begin{array}{l}
\mathbf{C} \\
\mathbf{A}
\end{array}\right) \rightarrow[r]
$$

be a colouring. Define $\bar{\chi}:\left(\begin{array}{c}\mathbf{C}_{0} \\ \operatorname{coll}(\mathbf{A})\end{array}\right) \rightarrow r$ by $\bar{\chi}\left(\mathbf{A}^{\prime}\right)=\chi\left(\operatorname{lift}\left(\mathbf{A}^{\prime}, \operatorname{recipe}(\mathbf{A})\right)\right.$ for any $\mathbf{A}^{\prime} \in$ $\left(\begin{array}{c}\mathbf{C}_{0} \\ \operatorname{coll}(\mathbf{A})\end{array}\right)$. Then there exists $\mathbf{B}^{\prime} \in\left(\begin{array}{c}\mathbf{C}_{0} \\ \operatorname{coll}(\mathbf{B})\end{array}\right)$ such that $\bar{\chi}$ is constant on $\left(\begin{array}{c}\mathbf{B}^{\prime} \\ \operatorname{coll}(\mathbf{A})\end{array}\right)$. Note that $\mathbf{B}^{\prime \prime}=\operatorname{lift}\left(\mathbf{B}^{\prime}, \operatorname{recipe}(\mathbf{B})\right) \in\left(\begin{array}{l}\mathbf{C} \\ \mathbf{B}\end{array}\right)$. 
Now for $\mathbf{A}^{\prime}, \mathbf{A}^{\prime \prime} \in\left(\begin{array}{c}\mathbf{B}^{\prime \prime} \\ \mathbf{A}\end{array}\right)$, we obtain by Lemma 4.1:

$$
\begin{aligned}
\chi\left(\mathbf{A}^{\prime}\right) & =\chi\left(\operatorname{lift}\left(\operatorname{coll}\left(\mathbf{A}^{\prime}\right), \operatorname{recipe}(\mathbf{A})\right)\right. \\
& =\bar{\chi}\left(\operatorname{coll}\left(\mathbf{A}^{\prime}\right)\right) \\
& =\bar{\chi}\left(\operatorname{coll}\left(\mathbf{A}^{\prime \prime}\right)\right) \\
& =\chi\left(\operatorname{lift}\left(\operatorname{coll}\left(\mathbf{A}^{\prime \prime}\right), \operatorname{recipe}(\mathbf{A})\right)\right. \\
& =\chi\left(\mathbf{A}^{\prime \prime}\right) .
\end{aligned}
$$

Similarly we can step up the expansion property, and again we provide the proof only for the case of $\mathbf{T}\left[I_{n}\right]$ as the others are similar.

Theorem 4.3. If Age $\left(\mathbf{T}^{*}\right)$ satisfies the expansion property with respect to Age $(\mathbf{T})$, then Age $\left(\mathbf{T}\left[I_{n}\right]^{*}\right)$ satisfies the expansion property with respect to Age $\left(\mathbf{T}\left[I_{n}\right]\right)$.

Proof. Consider $\mathbf{A} \in \operatorname{Age}\left(\mathbf{T}\left[I_{n}\right]\right)$. Then $\overline{\mathbf{A}}=\operatorname{coll}(\mathbf{A}) \in \operatorname{Age}(\mathbf{T})$ and by assumption we can find a structure $\overline{\mathbf{B}} \in \operatorname{Age}(\mathbf{T})$ such that any $\mathbf{T}^{*}$ expansion of $\overline{\mathbf{A}}$ embeds in any $\mathbf{T}^{*}$ expansion of $\overline{\mathbf{B}}$. We claim that $\overline{\mathbf{B}}\left[I_{n}\right] \in \operatorname{Age}\left(\mathbf{T}\left[I_{n}\right]\right)$ is is the required structure.

This is because any $\mathbf{T}\left[I_{n}\right]^{*}$ expansion $\mathbf{A}^{*}$ of $\mathbf{A}$ is of the form $\operatorname{lift}\left(\overline{\mathbf{A}}^{*}, \sigma\right)$, for some recipe $\sigma$ and some $\mathbf{T}^{*}$ expansion $\overline{\mathbf{A}}^{*}$ of $\overline{\mathbf{A}}$. Similarly any $\mathbf{T}\left[I_{n}\right]^{*}$ expansion $\mathbf{B}^{*}$ of $\overline{\mathbf{B}}\left[I_{n}\right]$ is of the form $\operatorname{lift}\left(\overline{\mathbf{B}}^{*}, \alpha\right)$, for some recipe $\alpha$ and some $\mathbf{T}^{*}$ expansion $\overline{\mathbf{B}}^{*}$ of $\overline{\mathbf{B}}$. Since $\overline{\mathbf{A}}^{*}$ embeds in $\overline{\mathbf{B}}^{*}$, it is easily seen that $\mathbf{A}^{*}$ embeds into $\mathbf{B}^{*}$.

We state the expansion property for the other cases for reference.

Theorem 4.4. If Age $\left(\mathbf{T}^{*}\right)$ satisfies the expansion property with respect to Age $(\mathbf{T})$, then $\operatorname{Age}\left(\hat{\mathbf{T}}^{*}\right)$ satisfies the expansion property with respect to Age $(\hat{\mathbf{T}})$.

\section{$5 \mathbf{T}\left[\boldsymbol{I}_{\omega}\right]$}

In this section, we treat the case of $\mathbf{T}\left[I_{\omega}\right]$ separately; the reason is that the unary predicable $L_{i}$ are not needed here so extra care must be taken. We first deal with the case when $\mathbf{T}$ is an infinite homogeneous tournament, and the structure $\mathbf{T}\left[I_{\omega}\right]$ is defined in the same way as $\mathbf{T}\left[I_{n}\right]$ for $n$ finite: it is the homogeneous directed graph whose vertex set consists of pairs $(x, i)$ with $x \in T$ and $i \in \mathbb{N}$. A pair $((x, i),(y, j))$ is an edge of $\mathbf{T}\left[I_{\omega}\right]$ if and only if $(x, y)$ is an edge of $\mathbf{T}$.

The structure $\mathbf{T}\left[I_{\omega}\right]^{*}$ is also defined in the same way as $\mathbf{T}\left[I_{n}\right]^{*}$ was from $\mathbf{T}^{*}$, except that the linear ordering is the lexicographical product of the ordering $<^{*}$ of $\mathbf{T}^{*}$ with an ordering $\prec$ on $\mathbb{N}$ that makes $(\mathbb{N}, \prec)$ isomorphic to $\mathbb{Q}$, and that as mentioned no unary predicate $L_{i}$ is added. Note that starting from a finite substructure $\overline{\mathbf{A}}$ of $\mathbf{T}^{*}$ and from a positive integer $n$, the same procedure yields a finite substructure $\overline{\mathbf{A}}\left[I_{n}\right]^{*}$ of $\mathbf{T}\left[I_{\omega}\right]^{*}$.

Suppose now $\mathbf{A}$ is a finite substructure of $\mathbf{T}\left[I_{\omega}\right]^{*}$. Define as before the collapse $\operatorname{coll}(\mathbf{A}) \subseteq \mathbf{T}$ as the projection on its first coordinates. Enumerating $\operatorname{coll}(\mathbf{A})=x_{1}<$ $\cdots<x_{k}$ using the ordering on $\mathbf{T}^{*}$, define the signature of $\mathbf{A}$ to be $\sigma=\left(a_{1}, \ldots, a_{k}\right)$ where $a_{i}=\left|A \cap\left\{\left(x_{i}, j\right): j \in \mathbb{N}\right\}\right|$.

We have the following: 
Lemma 5.1. Let $\mathbf{A}$ be a finite substructure of $\mathbf{T}\left[I_{\omega}\right]^{*}$ and $\overline{\mathbf{A}}=\operatorname{coll}(\mathbf{A})$ (so that $\overline{\mathbf{A}} \subseteq \mathbf{T}^{*}$ ). Then for any $r$ and any $n$, there is an $N \in \mathbb{N}$ such that for any colouring

$$
\chi:\left(\begin{array}{c}
\overline{\mathbf{A}}\left[I_{N}\right]^{*} \\
\mathbf{A}
\end{array}\right) \rightarrow[r]
$$

there exists $\mathbf{A}^{\prime} \in\left(\begin{array}{c}\overline{\mathbf{A}}\left[I_{N}\right]^{*} \\ \overline{\mathbf{A}}\left[I_{n}\right]^{*}\end{array}\right)$ such that $\chi$ is monochromatic on $\left(\begin{array}{c}\mathbf{A}^{\prime} \\ \mathbf{A}\end{array}\right)$.

Proof. Let $\sigma=\left(a_{1}, \ldots, a_{k}\right)$ be the signature of A. By Lemma 3.2, there exists $N$ such that

$$
N \rightarrow(n)_{r}^{\left(a_{1}, \ldots, a_{k}\right)} .
$$

The result follows from the fact that there is a one-to-one relationship between $\left(\begin{array}{c}\overline{\mathbf{A}}\left[I_{N}\right]^{*} \\ \mathbf{A}\end{array}\right)$ and $\left(\begin{array}{c}N, \ldots, N \\ a_{1}, \ldots, a_{k}\end{array}\right)$.

Using the preceding Lemma, we can thus guarantee that the colour of copies of $\mathbf{A}$ depends only on their collapse.

Corollary 5.2. Let $\mathbf{A}$ be a finite substructure of $\mathbf{T}\left[I_{\omega}\right]^{*}$, and $\overline{\mathbf{C}}$ be a finite substructure of $\mathbf{T}^{*}$. For any $r$ and any $n \in \mathbb{N}$, there is an $N \in \mathbb{N}$ such that for any colouring

$$
\chi:\left(\begin{array}{c}
\overline{\mathbf{C}}\left[I_{N}\right]^{*} \\
\mathbf{A}
\end{array}\right) \rightarrow r
$$

there exists $\mathbf{C}^{\prime} \in\left(\begin{array}{l}\overline{\mathbf{C}}\left[I_{N}\right]^{*} \\ \overline{\mathbf{C}}\left[I_{n}\right]^{*}\end{array}\right)$ such that for any $\overline{\mathbf{A}} \in\left(\begin{array}{c}\overline{\mathbf{C}} \\ \operatorname{coll}(\mathbf{A})\end{array}\right)$, $\chi$ is monochromatic on

$$
\left(\begin{array}{c}
\mathbf{C}^{\prime} \cap \overline{\mathbf{A}}\left[I_{N}\right]^{*} \\
\mathbf{A}
\end{array}\right) .
$$

Proof. First enumerate $\left(\begin{array}{c}\overline{\mathbf{C}} \\ \operatorname{coll}(\mathbf{A})\end{array}\right)=\overline{\mathbf{A}}_{\mathbf{1}}, \ldots, \overline{\mathbf{A}}_{\ell}$. Now let $N_{0}=n$, and for $i<\ell$ inductively use Lemma 5.1 to find $N_{i+1}$ so that there exists $\mathbf{A}^{\prime} \in\left(\begin{array}{c}\overline{\mathbf{A}}_{\mathbf{i}}\left[I_{N_{i+1}}\right]^{*} \\ \overline{\mathbf{A}}_{\mathbf{i}}\left[I_{N_{i}}\right]^{*}\end{array}\right)$ such that $\chi$ is monochromatic on $\left(\begin{array}{c}\mathbf{A}^{\prime} \\ \mathbf{A}\end{array}\right)$. Finally, set $N=N_{\ell}$.

Equivalently, if $\sigma=\left(a_{1}, \ldots, a_{k}\right)$ is the signature of $\mathbf{A}$, then for $i<\ell$ choose $N_{i+1}$ so that $N_{i+1} \rightarrow\left(N_{i}\right)_{r}^{\left(a_{1}, \ldots, a_{k}\right)}$ and let $N=N_{\ell}$.

We are now ready to show that $\operatorname{Age}\left(\mathbf{T}\left[I_{\omega}\right]^{*}\right)$ satisfies the Ramsey property, assuming $\mathbf{T}^{*}$ satisfies it in the first place.

Remark 5.3. It came to attention of the authors that one can also obtain the following result by applying Theorem 1.3 from [S13-3].

Theorem 5.4. The class Age $\left(\mathbf{T}\left[I_{\omega}\right]^{*}\right)$ satisfies the Ramsey property. 
Proof. We need to show that if $\mathbf{A} \subseteq \mathbf{B}$ are finite substructures of $\mathbf{T}\left[I_{\omega}\right]^{*}$, then for any $r$, there is a finite $\mathbf{C} \subseteq \mathbf{T}\left[I_{\omega}\right]^{*}$ such that

$$
\mathbf{C} \rightarrow(\mathbf{B})_{r}^{\mathbf{A}}
$$

Without loss of generality we may suppose that $\mathbf{B}=\overline{\mathbf{B}}\left[I_{n}\right]^{*}$ where $\overline{\mathbf{B}}$ is a finite substructure of $\mathbf{T}^{*}$.

Since Age $\left(\mathbf{T}^{*}\right)$ satisfies the Ramsey property, let $\overline{\mathbf{C}}$ be a finite substructure of $\mathbf{T}^{*}$ such that

$$
\overline{\mathbf{C}} \rightarrow(\overline{\mathbf{B}})_{r}^{\operatorname{coll}(\mathbf{A})} \text {. }
$$

By Corollary 5.2, there is $N$ and $\mathbf{C}^{\prime} \in\left(\begin{array}{l}\overline{\mathbf{C}}\left[I_{N}\right]^{*} \\ \overline{\mathbf{C}}\left[I_{n}\right]^{*}\end{array}\right)$ such that $\chi$ is monochromatic on all copies of $\mathbf{A}$ in $\mathbf{C}^{\prime}$ having the same collapse.

Thus define $\bar{\chi}:\left(\begin{array}{c}\overrightarrow{\mathbf{C}} \\ \operatorname{coll}(\mathbf{A})\end{array}\right) \rightarrow[r]$ by $\bar{\chi}(\overline{\mathbf{A}})=\chi\left(\mathbf{A}^{\prime}\right)$ for some (any) $\mathbf{A}^{\prime} \in\left(\begin{array}{l}\mathbf{C}^{\prime} \\ \mathbf{A}\end{array}\right)$ such that $\operatorname{coll}\left(\mathbf{A}^{\prime}\right)=\overline{\mathbf{A}}$.

This is well defined by construction, and now by assumption there is $\tilde{\mathbf{B}} \in\left(\begin{array}{l}\overline{\mathbf{C}} \\ \overline{\mathbf{B}}\end{array}\right)$ such that $\bar{\chi}$ is monochromatic on $\left(\begin{array}{c}\tilde{\mathbf{B}} \\ \operatorname{coll}(\mathbf{A})\end{array}\right)$. Finally set

$$
\mathbf{B}^{\prime}=\tilde{\mathbf{B}}\left[I_{N}\right]^{*} \cap \mathbf{C}^{\prime} .
$$

Since $\mathbf{C}^{\prime} \cong \overline{\mathbf{C}}\left[I_{n}\right]^{*}$, we have $\mathbf{B}^{\prime} \cong \overline{\mathbf{B}}\left[I_{n}\right]^{*}=\mathbf{B}$.

Now for $\mathbf{A}^{\prime}, \mathbf{A}^{\prime \prime} \in\left(\begin{array}{c}\mathbf{B}^{\prime} \\ \mathbf{A}\end{array}\right)$, we have $\operatorname{coll}\left(\mathbf{A}^{\prime}\right), \operatorname{coll}\left(\mathbf{A}^{\prime \prime}\right) \in\left(\begin{array}{c}\tilde{\mathbf{B}} \\ \operatorname{coll}(\mathbf{A})\end{array}\right)$, and therefore:

$$
\chi\left(\mathbf{A}^{\prime}\right)=\bar{\chi}\left(\operatorname{coll}\left(\mathbf{A}^{\prime}\right)\right)=\bar{\chi}\left(\operatorname{coll}\left(\mathbf{A}^{\prime \prime}\right)\right)=\chi\left(\mathbf{A}^{\prime \prime}\right) .
$$

This completes the proof.

Theorem 5.5. If Age $\left(\mathbf{T}^{*}\right)$ satisfies the expansion property with respect to Age $(\mathbf{T})$, then $\operatorname{Age}\left(\mathbf{T}\left[I_{\omega}\right]^{*}\right)$ satisfies the expansion property with respect to $\operatorname{Age}\left(\mathbf{T}\left[I_{\omega}\right]\right)$.

Proof. Suppose $\mathbf{A} \in \operatorname{Age}\left(\mathbf{T}\left[I_{\omega}\right]\right)$. It embeds in $\overline{\mathbf{A}}\left[I_{n}\right]$ for some $n$, where $\overline{\mathbf{A}}=\operatorname{coll}(\mathbf{A}) \in$ Age $(\mathbf{T})$. Find $\overline{\mathbf{B}} \in \operatorname{Age}(\mathbf{T})$ such that any expansion of $\overline{\mathbf{A}}$ embeds in any expansion of $\overline{\mathbf{B}}$. When expanding $\mathbf{A}$, each part is linearly ordered. Therefore, the convexly ordered expansion $\mathbf{A}^{*}$ must embed in any expansion $\overline{\mathbf{B}}\left[I_{n}\right]^{*}$ (recall we omitted the relations $L_{i}$ ).

If $\mathbf{T}$ is finite, then only the case $\mathbf{T}=C_{3}$ needs attention since the case $\mathbf{T}=I_{1}$ is trivial. So let $\mathbf{T}=C_{3}$ and expand $\mathbf{T}\left[I_{\omega}\right]$ to $\mathbf{T}\left[I_{\omega}\right]^{*}$ as follows: We can assume the universe is $T=[3]$, and let $P_{0}, \ldots, P_{2}$ be unary predicates and $<$ be a binary predicate. The expansion $\mathbf{T}\left[I_{\omega}\right]^{*}$ is obtained by interpreting $P_{i}=\{(i, j):(i, j) \in \mathbb{N}\}$ for $i \in[3]$, and $<^{*}$ as a dense linear order on each $P_{i}$ such that $P_{0}<{ }^{*} P_{1}<{ }^{*} P_{2}$.

Theorem 5.6. Age $\left(C_{3}\left[I_{\omega}\right]^{*}\right)$ satisfies the Ramsey property.

Proof. A finite structure $\mathbf{A}^{*} \in \operatorname{Age}\left(C_{3}\left[I_{\omega}\right]^{*}\right)$ can be viewed as an element in $\left(\begin{array}{c}\mathbb{N}, \mathbb{N}, \mathbb{N} \\ a_{1}, a_{2}, a_{3}\end{array}\right)$. Therefore, the Ramsey property follows from Lemma 3.2.

Similarly the expansion property is immediate.

Theorem 5.7. Age $\left(C_{3}\left[I_{\omega}\right]^{*}\right)$ satisfies the expansion property with respect to $\operatorname{Age}\left(C_{3}\left[I_{\omega}\right]\right)$.

And this also completes our program verification in this case. 


\section{$6 \quad I_{n}[\mathrm{~T}]$}

We again treat the case with $n$ finite separately. For $\mathbf{T}$ be a countable homogeneous tournament, the structure $I_{n}[\mathbf{T}]$ is then the disjoint union of $n$ copies of $\mathbf{T}$. We define the structure $I_{n}[\mathbf{T}]^{*}$ as the disjoint union of $n$ copies of $\mathbf{T}^{*}$, for which each copy of $\mathbf{T}^{*}$ corresponds to a unary predicate, and which is equipped with a convex linear order that orders the parts in a given specific pattern. More formally, the universe of $I_{n}[\mathbf{T}]^{*}$ is made of all pairs $(i, x)$ with $i \in[n]$ and $x \in T^{*}$. When $R$ is a relational symbol in $L_{\mathbf{T}^{*}} \backslash\{<\}$, set $R^{\mathbf{T}\left[I_{n}\right]^{*}}(\vec{u})$ iff $R^{\mathbf{T}^{*}}(\vec{x})$, where $\vec{u}=\left(\left(k, x_{1}\right), \ldots,\left(k, x_{n}\right)\right)$, for some $k$. As for $<$, interpret it as the lexicographical ordering of the usual order on $[n]$ and $<^{*}$ as per $\mathbf{T}^{*}$. Finally, add to the resulting structure $n$ new unary predicates $\left(P_{i}^{I_{n}[\mathbf{T}]^{*}}\right)_{i \in[n]}$ defined by $P_{i}^{I_{n}[\mathbf{T}]^{*}}(j, x)$ iff $j=i$. Note that therefore $P_{0}^{I_{n}[\mathbf{T}]^{*}}<{ }^{I_{n}[\mathbf{T}]^{*}} \cdots<^{I_{n}[\mathbf{T}]^{*}} P_{n-1}^{I_{n}[\mathbf{T}]^{*}}$. Note also that starting from a finite substructure $\overline{\mathbf{A}}$ of $\mathbf{T}^{*}$, the same procedure yields a finite substructure $I_{n}[\overline{\mathbf{A}}]^{*}$ of $I_{n}[\mathbf{T}]^{*}$.

Remark 6.1. The short proof of Theorem 6.2 is essentially the same as the standard proof of Lemma 3.2 and Theorem 2 from [S12]. We retain it here to keep the paper as self-contained as possible.

Theorem 6.2. The class Age $\left(I_{n}[\mathbf{T}]^{*}\right)$ satisfies the Ramsey Property.

Proof. Let $r$ be a positive integer and $\mathbf{A} \subseteq \mathbf{B} \subseteq I_{n}[\mathbf{T}]^{*}$, we need to find $\mathbf{C} \subseteq I_{n}[\mathbf{T}]^{*}$ such that

$$
\mathbf{C} \rightarrow(\mathbf{B})_{r}^{\mathbf{A}} .
$$

We proceed by induction on $n$, the base case $n=1$ simply being the Ramsey property of $\operatorname{Age}\left(\mathbf{T}^{*}\right)$.

For the induction step, suppose the desired property is true for $n-1$. First set $A_{i}=P_{i}^{I_{n}[T]^{*}} \cap A$ and $B_{i}=P_{i}^{I_{n}[T]^{*}} \cap B$. Note that

$$
\mathbf{A}^{0}:=\mathbf{A}_{0} \sqcup \ldots \sqcup \mathbf{A}_{n-2} \subseteq I_{n-1}[\mathbf{T}]^{*}
$$

and

$$
\mathbf{B}^{0}:=\mathbf{B}_{0} \sqcup \ldots \sqcup \mathbf{B}_{n-2} \subseteq I_{n-1}[\mathbf{T}]^{*}
$$

Let $\mathbf{C}^{1}$ be such that

$$
\mathbf{C}^{1} \rightarrow\left(\mathbf{B}_{n-1}\right) \underset{r}{\mid \mathbf{A}_{n-1}}\left(\begin{array}{l}
\mathbf{B}^{0} \\
\mathbf{A}^{0}
\end{array} \mid\right.
$$

Let $\mathbf{C}^{0}$ be such that

$$
\mathbf{C}^{0} \rightarrow\left(\mathbf{B}^{0}\right)_{r}^{\mathbf{A}^{0}}\left(_{\mathbf{A}_{n-1}}^{\mathbf{C}^{1}}\right) \mid \cdot
$$

Then $\mathbf{C}=\mathbf{C}^{0} \sqcup \mathbf{C}^{1}$ is as required. Indeed let

$$
\chi:\left(\begin{array}{l}
\mathbf{C} \\
\mathbf{A}
\end{array}\right) \rightarrow[r]
$$


be any colouring. Define

$$
\chi_{0}:\left(\begin{array}{c}
\mathbf{C}^{0} \\
\mathbf{A}^{0}
\end{array}\right) \rightarrow\left[r\left|\left(\begin{array}{c}
\left(\mathbf{A}_{n-1} \mathbf{C}^{1}\right. \\
\end{array}\right)\right|\right]
$$

by setting

$$
\chi_{0}\left(\mathbf{A}^{\prime}\right)=\left\{\left(\chi\left(\mathbf{A}^{\prime} \sqcup \mathbf{A}^{\prime \prime}\right), \mathbf{A}^{\prime \prime}\right): \mathbf{A}^{\prime \prime} \in\left(\begin{array}{c}
\mathbf{C}^{1} \\
\mathbf{A}_{n-1}
\end{array}\right)\right\}
$$

for $\mathbf{A}^{\prime} \in\left(\begin{array}{l}\mathbf{C}^{0} \\ \mathbf{A}^{0}\end{array}\right)$.

Subsequently, using a $\chi_{0^{-}}$-monochromatic $\mathbf{B}_{0} \in\left(\begin{array}{l}\mathbf{C}^{0} \\ \mathbf{B}^{0}\end{array}\right)$, further define

$$
\chi_{1}:\left(\begin{array}{c}
\mathbf{C}^{1} \\
\mathbf{A}_{n-1}
\end{array}\right) \rightarrow[r]
$$

by

$$
\chi_{1}\left(\mathbf{A}^{\prime \prime}\right)=\chi\left(\mathbf{A}^{\prime} \sqcup \mathbf{A}^{\prime \prime}\right)
$$

where $\mathbf{A}^{\prime \prime}$ is any member of $\left(\begin{array}{c}\mathbf{C}^{1} \\ \mathbf{A}_{n-1}\end{array}\right)$, for some $\mathbf{A}^{\prime} \in\left(\begin{array}{c}\mathbf{B}_{0} \\ \mathbf{A}^{0}\end{array}\right)$. This is independent of the choice of $\mathbf{A}^{\prime}$ since $\mathbf{B}_{0}$ is $\chi_{0}$-monochromatic.

Therefore, there is a $\chi_{1}$-monochromatic

$$
\mathbf{B}^{1} \in\left(\begin{array}{c}
\mathbf{C}^{1} \\
\mathbf{B}_{n-1}
\end{array}\right)
$$

and thus $\mathbf{B}_{0} \sqcup \mathbf{B}_{1}$ is $\chi$-monochromatic as desired.

Theorem 6.3. If Age $\left(\mathbf{T}^{*}\right)$ satisfies the expansion property with respect to Age $(\mathbf{T})$, then $\operatorname{Age}\left(I_{n}[\mathbf{T}]^{*}\right)$ satisfies the expansion property with respect to $\operatorname{Age}\left(I_{n}[\mathbf{T}]\right)$.

Proof. Suppose $\mathbf{A} \in \operatorname{Age}\left(I_{n}[\mathbf{T}]\right)$. Then $\mathbf{A}$ has the universe of the form $\{0\} \times A_{0} \sqcup \ldots \sqcup$ $\{n-1\} \times A_{n-1}$ with $\mathbf{A}_{i} \in \operatorname{Age}(\mathbf{T})$ or $A_{i}=\emptyset$. For every $i$, if $A_{i} \neq \emptyset$, find $\mathbf{B}_{i} \in \operatorname{Age}(\mathbf{T})$ such that any $\mathbf{T}^{*}$ expansion of $\mathbf{A}_{i}$ embeds in any $\mathbf{T}^{*}$ expansion of $\mathbf{B}_{i}$. If $A_{i}=\emptyset$, set $B_{i}=\emptyset$. We can assume that the universes $B_{i}$ are pairwise disjoint. Any $I_{n}[\mathbf{T}]^{*}$ expansion $\mathbf{A}^{*}$ of $\mathbf{A}$ has a universe of the form $\{0\} \times A_{f(0)}^{*} \sqcup \ldots \sqcup\{n-1\} \times A_{f(n-1)}^{*}$ for some bijection $f:[n] \rightarrow[n]$, where each $\mathbf{A}_{f(i)}^{*}$ is a $\mathbf{T}^{*}$ expansion of $\mathbf{A}_{i}$ and each $P_{i}^{\mathbf{A}^{*}}=A_{f(i)}^{*}$. Set $\mathbf{B}$ to be the convexly ordered structure in $\operatorname{Age}\left(I_{n}[\mathbf{T}]^{*}\right)$ with universe

$$
B=\{0\} \times\left(\bigcup_{i \in[n]} B_{i}\right) \sqcup \ldots \sqcup\{n-1\} \times\left(\bigcup_{i \in[n]} B_{i}\right)
$$

where the substructures with universes $\{k\} \times B_{i}$ are isomorphic to $\mathbf{B}_{i}$, for all $i$ and $k$. Therefore, the $I_{n}[\mathbf{T}]^{*}$ expansion $\mathbf{A}^{*}$ must embed in any $I_{n}[\mathbf{T}]^{*}$ expansion $\mathbf{B}^{*}$. 


\section{$7 \quad I_{\omega}[\mathrm{T}]$}

For $\mathbf{T}$ a countable homogeneous tournament, the structure $I_{\omega}[\mathbf{T}]$ is the disjoint union of countably many copies of $\mathbf{T}$. Formally, its universe is $\mathbb{N} \times T$, and $((i, x)(j, y))$ is an edge in $I_{\omega}[\mathbf{T}]$ iff $i=j$ and $(x, y)$ is an edge in $\mathbf{T}$. The structure $I_{\omega}[\mathbf{T}]^{*}$ is defined in the same way as $I_{n}[\mathbf{T}]^{*}$ was from $\mathbf{T}^{*}$, except that the linear ordering is the lexicographical product of a dense linear order $\prec$ on $\mathbb{N}$ with the ordering $<^{*}$ of $\mathbf{T}^{*}$. Here, no unary relation $P_{i}$ is added. Note also that starting from a finite substructure $\overline{\mathbf{A}}$ of $\mathbf{T}^{*}$ and from a positive integer $n$, the same procedure yields a finite substructure $I_{n}[\overline{\mathbf{A}}]^{*}$ of $I_{\omega}[\mathbf{T}]^{*}$.

For $i \in \mathbb{N}$, define the part $X_{i}$ to be the set of all pairs $(i, x)$ for $x \in \mathbf{T}$. For any $\mathbf{A} \subseteq I_{\omega}[\mathbf{T}]^{*}$, define the collapse coll $(\mathbf{A})$ to be the set $\left\{i: A \cap X_{i} \neq \emptyset\right\}$. If $\overline{\mathbf{A}} \subseteq \mathbf{T}^{*}$ and $\sigma$ is a finite subset of $\omega$, then we define $\sigma[\overline{\mathbf{A}}]^{*}$ to be the substructure of $I_{\omega}[\mathbf{T}]^{*}$ consisting of $(i, x)$ with $x \in \overline{\mathbf{A}}$ and $i \in \sigma$. Define $\left(\begin{array}{l}\mathbf{B} \\ \mathbf{A}\end{array}\right)_{\tau}$ to be the subset of $\left(\begin{array}{l}\mathbf{B} \\ \mathbf{A}\end{array}\right)$ consisting of those copies of $\mathbf{A}$ whose collapse is precisely $\tau$.

The following is a direct consequence of Theorem 6.2.

Lemma 7.1. Let $\mathbf{A}$ and $I_{m}[\overline{\mathbf{B}}]^{*}$ be a finite substructures of $I_{\omega}[\mathbf{T}]^{*}$ and let $\tau$ be a subset of $[m]$ of size $|\operatorname{coll}(\mathbf{A})|$. For any $r \geqslant 2$, there is a finite $\overline{\mathbf{C}} \subseteq \mathbf{T}^{*}$ such that for any colouring

$$
\chi:\left(\begin{array}{c}
I_{m}[\overline{\mathbf{C}}]^{*} \\
\mathbf{A}
\end{array}\right) \rightarrow[r]
$$

there exists $\mathbf{B}^{\prime} \in\left(\begin{array}{c}I_{m}[\overline{\mathbf{C}}]^{*} \\ I_{m}[\overline{\mathbf{B}}]^{*}\end{array}\right)$ such that $\left|\chi^{\prime \prime}\left(\begin{array}{c}\mathbf{B}^{\prime} \\ \mathbf{A}\end{array}\right)_{\tau}\right|=1$.

We symbolize this by

$$
I_{m}[\overline{\mathbf{C}}]^{*} \rightarrow\left(I_{m}[\overline{\mathbf{B}}]^{*}\right)_{r}^{\mathbf{A}, \tau}
$$

An iteration of the above argument yields the following.

Corollary 7.2. Let $\mathbf{A}$ and $I_{m}[\overline{\mathbf{B}}]^{*}$ be a finite substructures of $I_{\omega}[\mathbf{T}]^{*}$. For any $r \geqslant 2$, there is a finite $\overline{\mathbf{C}} \subseteq \mathbf{T}^{*}$ such that for any colouring

$$
\chi:\left(\begin{array}{c}
I_{m}[\overline{\mathbf{C}}]^{*} \\
\mathbf{A}
\end{array}\right) \rightarrow[r]
$$

there exists $\mathbf{B}^{\prime} \in\left(\begin{array}{c}I_{m}[\overline{\mathbf{C}}]^{*} \\ I_{m}[\overline{\mathbf{B}}]^{*}\end{array}\right)$ such that $\left|\chi^{\prime \prime}\left(\begin{array}{c}\mathbf{B}^{\prime} \\ \mathbf{A}\end{array}\right)_{\tau}\right|=1$ for any $\tau \in\left(\begin{array}{c}m \\ \operatorname{coll}(\mathbf{A})\end{array}\right)$.

We symbolize this by

$$
I_{m}[\overline{\mathbf{C}}]^{*} \rightarrow\left(I_{m}[\overline{\mathbf{B}}]^{*}\right)_{r}^{\mathbf{A}, \text { coll }}
$$

Proof. First enumerate the set $\left(\begin{array}{c}m \\ \operatorname{coll}(\mathbf{A})\end{array}\right)$ of $|\operatorname{coll}(\mathbf{A})|$-subsets of $[m]$ as $\tau_{1}, \ldots, \tau_{\ell}$. Let $\overline{\mathbf{C}}_{1}$ be such that

$$
I_{m}\left[\overline{\mathbf{C}}_{1}\right]^{*} \rightarrow\left(I_{m}[\overline{\mathbf{B}}]^{*}\right)_{r}^{\mathbf{A}, \tau_{1}}
$$

and for $i<\ell$ inductively use Lemma 7.1 to find $\overline{\mathbf{C}}_{i+1}$ such that

$$
I_{m}\left[\overline{\mathbf{C}}_{i+1}\right]^{*} \rightarrow\left(I_{m}\left[\overline{\mathbf{C}}_{i}\right]^{*}\right)_{r}^{\mathbf{A}, \tau_{i+1}}
$$

Finally, set $\overline{\mathbf{C}}=\overline{\mathbf{C}}_{\ell}$. 
Remark 7.3. As before, it came to attention of the authors that, one can also obtain the following result, by applying Theorem 1.4 from [S13-3].

Theorem 7.4. The class Age $\left(I_{\omega}[\boldsymbol{T}]^{*}\right)$ satisfies the Ramsey Property.

Proof. Let $r \in \mathbb{N}$ be positive and let $\mathbf{A} \subseteq \mathbf{B} \subseteq I_{\omega}[\mathbf{T}]^{*}$. We need to find $\mathbf{C} \subseteq I_{\omega}[\mathbf{T}]^{*}$ such that

$$
\mathbf{C} \rightarrow(\mathbf{B})_{r}^{\mathbf{A}} .
$$

Without loss of generality, we may suppose that $\mathbf{B}=I_{m}[\overline{\mathbf{B}}]^{*}$. If $|\operatorname{coll}(\mathbf{A})|=k$, then by Ramsey's theorem there is $N$ such that

$$
N \rightarrow(m)_{r}^{k}
$$

By Corollary 7.2, there is a finite $\overline{\mathbf{C}} \subseteq \mathbf{T}^{*}$ such that

$$
I_{N}[\overline{\mathbf{C}}]^{*} \rightarrow\left(I_{N}[\overline{\mathbf{B}}]^{*}\right)_{r}^{\mathbf{A}, \text { coll }}
$$

Let $\chi:\left(\begin{array}{c}I_{N}[\overline{\mathbf{B}}]^{*} \\ \mathbf{A}\end{array}\right) \rightarrow[r]$ be any colouring. By the above, we can find $\mathbf{B}^{\prime} \in\left(\begin{array}{l}I_{N}[\overline{\mathbf{C}}]^{*} \\ I_{N}[\overline{\mathbf{B}}]^{*}\end{array}\right)$ such that for any $\tau \in\left(\begin{array}{c}m \\ \operatorname{coll}(\mathbf{A})\end{array}\right)$, we have $\left|\chi^{\prime \prime}\left(\begin{array}{c}\mathbf{B}^{\prime} \\ \mathbf{A}\end{array}\right)_{\tau}\right|=1$.

Define $\bar{\chi}:\left(\begin{array}{c}N \\ \operatorname{coll}(\mathbf{A})\end{array}\right) \rightarrow[r]$ as follows: If $\tau \in\left(\begin{array}{c}N \\ \operatorname{coll}(\mathbf{A})\end{array}\right)$, set $\bar{\chi}(\tau)=\chi\left(\mathbf{A}^{\prime}\right)$ for some (any) $\mathbf{A}^{\prime} \in\left(\begin{array}{c}\mathbf{B}^{\prime} \\ \mathbf{A}\end{array}\right)_{\tau}$. The colouring $\bar{\chi}$ is well defined by $(*)$. Now, we can find $\sigma \in\left(\begin{array}{c}N \\ m\end{array}\right)$ such that $\bar{\chi}$ is monochromatic on $\left(\begin{array}{c}\sigma \\ \operatorname{coll}(\mathbf{A})\end{array}\right)$. Set

$$
\mathbf{B}^{\prime \prime}=\left\{(i, x) \in I_{\omega}[\mathbf{T}]^{*}: i \in \sigma\right\} \cap \mathbf{B}^{\prime} .
$$

Since $\mathbf{B}^{\prime} \cong I_{N}[\overline{\mathbf{B}}]^{*}$, we have $\mathbf{B}^{\prime \prime} \cong I_{m}[\overline{\mathbf{B}}]^{*}$. Let $\mathbf{A}^{\prime}, \mathbf{A}^{\prime \prime} \in\left(\begin{array}{c}\mathbf{B}^{\prime \prime} \\ \mathbf{A}\end{array}\right)$. Thus, $\operatorname{coll}\left(\mathbf{A}^{\prime}\right), \operatorname{coll}\left(\mathbf{A}^{\prime \prime}\right) \in$ $\left(\begin{array}{c}\sigma \\ |\operatorname{coll}(\mathbf{A})|\end{array}\right)$. Therefore,

$$
\chi\left(\mathbf{A}^{\prime}\right)=\bar{\chi}\left(\operatorname{coll}\left(\mathbf{A}^{\prime}\right)\right)=\bar{\chi}\left(\operatorname{coll}\left(\mathbf{A}^{\prime \prime}\right)\right)=\chi\left(\mathbf{A}^{\prime \prime}\right)
$$

which completes the proof.

Theorem 7.5. If Age $\left(\mathbf{T}^{*}\right)$ satisfies the expansion property with respect to Age $(\mathbf{T})$, then $\operatorname{Age}\left(I_{\omega}[\mathbf{T}]^{*}\right)$ satisfies the expansion property with respect to $\operatorname{Age}\left(I_{\omega}[\mathbf{T}]\right)$.

Proof. Suppose $\mathbf{A} \in \operatorname{Age}\left(I_{\omega}[\mathbf{T}]\right)$. Without loss of generality, we can assume that $\mathbf{A}$ has the universe of the form $\{0\} \times A_{0} \sqcup \ldots \sqcup\{n-1\} \times A_{n-1}$ with $\mathbf{A}_{i} \in \operatorname{Age}(\mathbf{T})$. For every $i$, find $\mathbf{B}_{i} \in$ Age $(\mathbf{T})$ such that any expansion of $\mathbf{A}_{i}$ embeds in any expansion of $\mathbf{B}_{i}$, and we can assume that the universes $B_{i}$ are pairwise disjoint. Further, we can assume that any expansion $\mathbf{A}^{*}$ of $\mathbf{A}$ has the universe of the form $\{0\} \times A_{f(0)}^{*} \sqcup \ldots \sqcup\{n-1\} \times A_{f(n-1)}^{*}$ for some bijection $f:[n] \rightarrow[n]$, where each $\mathbf{A}_{f(i)}^{*}$ is an expansion of $\mathbf{A}_{i}$ and $A_{f(i)}^{*}<A_{f(j)}^{*}$ for $i<j$. Set $\mathbf{B}$ to be the convexly ordered structure in $\operatorname{Age}\left(I_{\omega}[\mathbf{T}]^{*}\right)$ with universe

$$
B=\{0\} \times\left(\bigcup_{i \in[n]} B_{i}\right) \sqcup \ldots \sqcup\{n-1\} \times\left(\bigcup_{i \in[n]} B_{i}\right)
$$


where the substructures with universes $\{k\} \times B_{i}$ are isomorphic to $\mathbf{B}_{i}$, for all $i$ and $k$. Moreover, we ensure that $(i, a)<(j, b)$ for $i<j$ and all $a, b \in B$. Therefore, the expansion $\mathbf{A}^{*}$ must embed in any expansion $\mathbf{B}^{*}$.

If $\mathbf{T}$ is finite, then again only the case $\mathbf{T}=C_{3}$ requires attention. Expand $I_{\omega}[\mathbf{T}]$ to $I_{\omega}[\mathbf{T}]^{*}$ as follows:

We can assume the universe is $T=[3]$, and let $L_{0}, \ldots, L_{2}$ be unary predicates and $<$ be a new binary predicate. The expansion $\mathbf{T}\left[I_{\omega}\right]^{*}$ is obtained by interpreting $L_{i}=\{(j, i)$ : $j \in \mathbb{N}\}$ for $i \in[3]$, and $<^{*}$ as a dense linear order on each $L_{i}$ such that $L_{0}<^{*} L_{1}<^{*} L_{2}$. We can immediately verify the following.

Lemma 7.6. Let $\boldsymbol{A}, \boldsymbol{A}^{\prime} \subseteq I_{\omega}[\mathbf{T}]^{*}$. Then $\boldsymbol{A}$ and $\boldsymbol{A}^{\prime}$ are isomorphic if and only if there is an order preserving bijection $f: \operatorname{coll}(\boldsymbol{A}) \rightarrow \operatorname{coll}\left(\boldsymbol{A}^{\prime}\right)$ such that for every $i,\{j:(i, j) \in$ $A\}=\left\{j:(f(i), j) \in A^{\prime}\right\}$.

From this we immediately obtain the following using the usual Ramsey Theorem.

Theorem 7.7. The class Age $\left(I_{\omega}\left[C_{3}\right]^{*}\right)$ satisfies the Ramsey property.

And similarly for the expansion property.

Theorem 7.8. The class $\operatorname{Age}\left(I_{\omega}\left[C_{3}\right]^{*}\right)$ satisfies the expansion property with respect to $\operatorname{Age}\left(I_{\omega}\left[C_{3}\right]\right)$.

Proof. Let $\mathbf{A} \in \operatorname{Age}\left(I_{\omega}\left[C_{3}\right]\right)$, then any expansion $\mathbf{A}^{*}$ of $\mathbf{A}$ will embed in any expansion of

$$
\{(i, j): i \in \operatorname{coll}(\mathbf{A}), j \in[3]\} .
$$

\section{Complete $n$-partite directed graphs}

For $n \leqslant \omega$, by a complete $n$-partite directed graph we mean a directed graph such that the non-edge relation $\perp$ is an equivalence relation with at most $n$ many classes. For each $n$, the class of all such graphs is a Fraïssé class. The corresponding limit is denoted $n * I_{\omega}$, and consists of $n$ many disjoint copies of $I_{\omega}$ between which edges are distributed in a complete and random way. For $n \in \mathbb{N}$ we construct the expansion $n * I_{\omega}^{*}$ by adding a convex linear ordering together with $n$ many unary relations $P_{i}^{n * I_{\omega}^{*}}$ corresponding to the parts of $n * I_{\omega}$ and so that $P_{0}^{n * I_{\omega}^{*}}<^{n * I_{\omega}^{*}} \ldots<^{n * I_{\omega}^{*}} P_{n-1}^{n * I_{\omega}^{*}}$. For $n=\omega$, construct $\omega * I_{\omega}^{*}$ by simply adding to $\omega * I_{\omega}$ a convex linear ordering that orders each part in a dense linear order, as well as the set of parts in a dense linear order; note that we do not add any unary predicates in this case. Those expansions are precompact, and we show that their ages have the Ramsey property and show the appropriate expansion properties.

If $\mathbf{B}$ is a complete $n$-partite directed graph, say that a subgraph is transversal when it has exactly one point in each $\perp$ equivalence class of $\mathbf{B}$. 
Remark 8.1. It came to the attention of the authors that there is a simpler proof of the lemmas below, based on the result in [S13-2]: One takes the output of the result of [S13-2] and prunes it, arriving at an element of the age of $n * I_{\omega}$. Though, we include the direct proof based on the partite construction, we will revisit the result of [S13-2] in section 9.

Lemma 8.2. Let $n, r \in \mathbb{N}$ be positive and suppose $\boldsymbol{B}$ is a finite substructure of $n * I_{\omega}^{*}$ with exactly $n$ parts. Suppose $\boldsymbol{A}$ is an n-partite directed subgraph of $\boldsymbol{B}$ which is transversal. Then there exists a finite $n$-partite graph $\boldsymbol{C}$ such that

$$
\boldsymbol{C} \rightarrow(\boldsymbol{B})_{r}^{\boldsymbol{A}}
$$

Proof. This is identical to the proof of the partite lemma in [NR89].

Suppose now that $\mathbf{A}$ is a directed subgraph of $\mathbf{B}$, and that the parts of $\mathbf{B}$ are written $Y_{i}$ with $i \in[n]$. Define the trace of $\mathbf{A}$ (in $\mathbf{B}$ ) to be

$$
\operatorname{tr}(\mathbf{A})=\left\{i: A \cap Y_{i} \neq \emptyset\right\} .
$$

For $x \in B$, define the trace of $x$ to be $\operatorname{tr}(x)=\operatorname{tr}(\{x\})$, thus $x \in Y_{\operatorname{tr}(x)}$.

We begin with the following special case of the Ramsey property.

Lemma 8.3. Let $n, r \in \mathbb{N}$ with $r>0$, suppose $\boldsymbol{A} \leqslant \boldsymbol{B}$ are finite substructure of $n * I_{\omega}^{*}$ with exactly $n$ parts each. Then there exists a finite $\boldsymbol{C} \subseteq n * I_{\omega}^{*}$ such that

$$
\boldsymbol{C} \rightarrow(\boldsymbol{B})_{r}^{\boldsymbol{A}}
$$

Proof. What follows is a modification of the partite construction in [NR89]. Let $\mathbf{A}$ and $\mathbf{B}$ be as above, and denote by $Y_{i}$, with $i<n$, the parts of $\mathbf{B}$. Set $a=|A|, b=|B|$, $a_{i}=\left|A \cap Y_{i}\right|$, and $b_{i}=\left|B \cap Y_{i}\right|$ for $i<n$. By Lemma 3.2, there exist disjoint $C_{i} \subseteq \mathbb{N}$ with $i<n$, such that

$$
\left(C_{0}, \ldots, C_{n-1}\right) \rightarrow\left(b_{0}, \ldots, b_{n-1}\right)_{r}^{\left(a_{0}, \ldots, a_{n-1}\right)} .
$$

We can assume that $C_{0}=\left\{1, \ldots,\left|C_{0}\right|\right\}$ and in general,

$$
C_{i}=\left\{\max \left(C_{i-1}\right)+1, \ldots, \max \left(C_{i-1}\right)+\left|C_{i}\right|\right\} .
$$

Set $p=\max \left(C_{n-1}\right)$. Enumerate

$$
\left(\begin{array}{c}
C_{0}, \ldots, C_{n-1} \\
a_{0}, \ldots, a_{n-1}
\end{array}\right)=\left\{N_{1}, \ldots, N_{\alpha}\right\}
$$

and

$$
\left(\begin{array}{c}
C_{0}, \ldots, C_{n-1} \\
b_{0}, \ldots, b_{n-1}
\end{array}\right)=\left\{M_{1}, \ldots, M_{\beta}\right\}
$$

Now for every $j \in[\beta], M_{j}$ is a disjoint union of $A_{k}^{j}$, s such that $A_{k}^{j}=M_{j} \cap C_{k}$, for every $k<n$. Construct a substructure $\mathbf{Q}^{0}$ of $n * I_{\omega}^{*}$ as follows: the vertex set $Q^{0}$ consists of all pairs $(i, j)$ such that $i \in M_{j}$. For each $j \in[\beta]$, reproduce a copy $\mathbf{B}_{j}$ of $\mathbf{B}$ with universe 
$\left\{(i, j): i \in M_{j}\right\}$, so that $P_{k}^{\mathbf{B}_{j}}=B_{j} \cap C_{k}$ (for every $\left.k<n\right)$ and $(i, j)<^{\mathbf{B}_{j}}\left(i^{\prime}, j\right)$ if and only if $i<i^{\prime}$. To complete our set of edges, for every $i<i^{\prime}$ and $j<j^{\prime}$, if $i \in A_{k}^{j}$ and $i^{\prime} \in A_{k^{\prime}}^{j^{\prime}}$ where $k<k^{\prime}$, then we add $\left((i, j),\left(i^{\prime}, j^{\prime}\right)\right)$. For every $k \in[n], P_{k}^{\mathbf{Q}^{0}}$ is the disjoint union of $P_{k}^{\mathbf{B}_{j}}$ s. Moreover, we set each non-structural part to be

$$
\mathcal{M}_{i}=\{(i, j): j \in[\beta]\}
$$

for $i \in[p]$. Finally, add a linear ordering (convex with respect to both partitions) that extends the linear orderings that already exist on each copy of $\mathbf{B}$ : If $j<j^{\prime}$, then set $(i, j)<\mathbf{Q}^{0}\left(i, j^{\prime}\right)$. If $i<i^{\prime}, j \neq j^{\prime}$, then set $(i, j)<\mathbf{Q}^{0}\left(i^{\prime}, j^{\prime}\right)$. The resulting structure is $\mathbf{Q}^{0}$.

Next, we construct a sequence $\mathbf{Q}^{1}, \ldots, \mathbf{Q}^{\alpha}$ inductively using lemma 8.3, as in [NR89]. To construct $\mathbf{Q}^{i}$ from $\mathbf{Q}^{i-1}$, we first consider the directed subgraph of $\mathbf{Q}^{i-1}$ whose trace is $N^{i-1}$ with respect to the non-structural partition, call it $\mathbf{H}^{i-1}$. Applying lemma 8.3, we get an $a$-partite $\mathbf{G}^{i-1}$ such that

$$
\mathbf{G}^{i-1} \rightarrow\left(\mathbf{H}^{i-1}\right)_{r}^{\mathbf{A}}
$$

Subsequently, we expand each copy of $\mathbf{H}^{i-1}$ to a copy of $\mathbf{Q}^{i-1}$, and add a convex (with respect to the structural partition) linear ordering that extends all the linear orderings that already exist on each copy of $\mathbf{Q}^{i-1}$. We call the result $\mathbf{Q}^{i}$. Following this procedure, the directed graph induced by the structure $\mathbf{Q}^{\alpha}$ is not complete with respect to the structural partition, but it can be made complete by adding new edges. Let $\mathbf{C}$ be any structure obtained that way. We claim that $\mathbf{C}$ is as required.

Let $\chi:\left(\begin{array}{l}\mathbf{C} \\ \mathbf{A}\end{array}\right) \rightarrow[r]$ be any colouring. Then there will be $\mathbf{Q}^{\prime} \in\left(\begin{array}{c}\mathbf{C} \\ \mathbf{Q}^{0}\end{array}\right)$ such that the colouring of each copy of $\mathbf{A}$ depends only on its trace with respect to the non-structural partition. The conclusion follows from the construction of $\mathbf{Q}^{0}$ now using routine arguments.

We are now ready to prove the general result.

Theorem 8.4. The age of $n * I_{\omega}^{*}$ has the Ramsey property for each $n \in \mathbb{N}$.

Proof. Let $k \leqslant n, r \in \mathbb{N}$ with $r>0, \mathbf{B}$ a finite substructure of $n * I_{\omega}^{*}$ with exactly $n$ parts, and $\mathbf{A}$ is a substructure of $\mathbf{B}$ with $k$ parts. Then we will show that there exists a finite $\mathbf{C} \subseteq n * I_{\omega}^{*}$ with $n$ parts such that

$$
\mathbf{C} \rightarrow(\mathbf{B})_{r}^{\mathbf{A}}
$$

Note that because of the unary relations that are interpreted as the parts of $n * I_{\omega}^{*}$, all copies of $\mathbf{A}$ in $\mathbf{B}$ have same trace $\tau$. Let $\overline{\mathbf{B}}$ be the substructure of $\mathbf{B}$ induced by the points $v \in B$ with $\operatorname{tr}(v) \in \tau$. Apply Lemma 8.3 to find $\overline{\mathbf{C}}$ such that $\overline{\mathbf{C}} \rightarrow(\overline{\mathbf{B}})_{r}^{\mathbf{A}} . \sigma$ be the trace of $\mathbf{B}$. Now extend each copy of $\overline{\mathbf{B}}$ in $\overline{\mathbf{C}}$ to a copy of $\overline{\mathbf{B}}$, via amalgamation. Set $\mathbf{C}$ to be the result of this extension with appropriate edges and linear ordering added.

This result allows us to show the same is true for the infinite partite structure. 
Theorem 8.5. The age of $\omega * I_{\omega}^{*}$ has the Ramsey property.

Proof. Let $k \leqslant n, r \in \mathbb{N}$ with $r>0, \mathbf{B}$ a finite substructure of $\omega * I_{\omega}^{*}$ with $n$ parts, and $\mathbf{A}$ a substructure of $\mathbf{B}$ with $k$ parts.

Expand for the moment $\omega * I_{\omega}^{*}$ with unary predicates corresponding to its parts, calling the resulting structure $\omega * I_{\omega}^{* *}$. Using Ramsey's theorem, we can find $N$ such that $N \rightarrow$ $(n)_{r}^{k}$, and we enumerate the $n$-subsets of $[N]$ as $\tau_{1}, \ldots, \tau_{\ell}$. For each $i \leqslant l$ consider a copy $\mathbf{B}_{i}$ of $\mathbf{B}$ with trace $\tau_{i}$ in $\omega * I_{\omega}^{*}$ so that the $\mathbf{B}_{i}$ 's are pairwise disjoint. Set $\overline{\mathbf{B}}$ to be the substructure of $\omega * I_{\omega}^{*}$ supported by the union of these $\mathbf{B}_{i}$ 's.

Note that all copies of $\mathbf{A}$ with fixed trace in $\omega * I_{\omega}^{*}$ induce isomorphic structures in $\omega * I_{\omega}^{* *}$. Using Theorem 8.4, we can find a finite substructure $\mathbf{C}$ of $\omega * I_{\omega}^{*}$ such that every $r$-colouring $\chi$ of $\left(\begin{array}{l}\mathbf{C} \\ \mathbf{A}\end{array}\right)$, there exists a copy $\overline{\mathbf{B}}^{\prime} \in\left(\begin{array}{l}\mathbf{C} \\ \overline{\mathbf{B}}\end{array}\right)$ such that $\chi$ restricted to $\left(\begin{array}{c}\overline{\mathbf{B}}^{\prime} \\ \mathbf{A}\end{array}\right)$ depends only on the trace.

To complete the proof, define $\bar{\chi}:\left(\begin{array}{l}N \\ k\end{array}\right) \rightarrow[r]$ by setting $\bar{\chi}(\sigma)$ to be the colour of any copy of $\mathbf{A}$ in $\overline{\mathbf{B}}^{\prime}$ with trace $\sigma$; this is well defined by construction. By the choice of $N$, there is a $\bar{\chi}$-monochromatic $\tau_{i_{0}}$, and by construction, there is $\mathbf{B}^{\prime} \in\left(\begin{array}{c}\overline{\mathbf{B}}^{\prime} \\ \mathbf{B}\end{array}\right)$ with trace $\tau_{i_{0}}$, which completes the proof.

We now more to the expansion property, and note that the proof of the theorem below follows the template found in the proof of Lemma 5.2 in [N96] and the proofs of the expansion properties found in [S13-1].

Theorem 8.6. The age of $\omega * I_{\omega}^{*}$ satisfies the expansion property with respect to the age of $\omega * I_{\omega}$.

Proof. Let $\mathbf{A} \in \operatorname{Age}\left(\omega * I_{\omega}\right)$, and fix an arbitrary a convex linear ordering $<$ on $A$. We will produce a structure $\mathbf{B} \in \operatorname{Age}\left(\omega * I_{\omega}\right)$ such that $\mathbf{A}^{*}$ embeds in any expansion of $\mathbf{B}$.

Consider the following structures:

- $\mathbf{E}_{1}$ is the linearly ordered directed graph with universe $\{a, b\}$ and the directed edge $(a, b)$ such that $a<b$.

- $\mathbf{E}_{2}$ is the linearly ordered directed graph with universe $\{a, b\}$ and no edges such that $a<b$.

Set $<_{\text {rev }}$ to be the same as $<$ but with the ordering on each part (i.e., the ordering on non-edges) reversed. Set $>_{\text {rev }}$ to be the same as $>$ but with the ordering on each part reversed.

Now find $\mathbf{A}_{1} \in \operatorname{Age}\left(\omega * I_{\omega}^{*}\right)$ so that $(\mathbf{A},<),(\mathbf{A},>),\left(\mathbf{A},<_{\text {rev }}\right)$ and $\left(\mathbf{A},>_{\text {rev }}\right)$ embed in $\mathbf{A}_{1}$, and similarly find $\mathbf{A}_{2} \in \operatorname{Age}\left(\omega * I_{\omega}^{*}\right)$ so that $\mathbf{A}_{1}, \mathbf{E}_{1}$ and $\mathbf{E}_{2}$ all embed in $\mathbf{A}_{2}$.

Since $\operatorname{Age}\left(\omega * I_{\omega}^{*}\right)$ satisfies the Ramsey property, we can find $\mathbf{C}_{i} \in \operatorname{Age}\left(\omega * I_{\omega}^{*}\right)$ so that $\mathbf{C}_{1} \rightarrow\left(\mathbf{A}_{2}\right)_{2}^{\mathbf{E}_{1}}$ and $\mathbf{C}_{2} \rightarrow\left(\mathbf{C}_{1}\right)_{2}^{\mathbf{E}_{2}}$.

Let $<^{\prime}$ be the ordering on $\mathbf{C}_{2}$, and $\prec$ be any convex linear ordering on $C_{2}$, and consider the following colouring on ordered pairs in the universe of $\mathbf{C}_{2}$ :

$$
\chi((u, v))= \begin{cases}0 & \text { if the two orders }<^{\prime} \text { and } \prec \text { agree on }\{u, v\} . \\ 1 & \text { otherwise }\end{cases}
$$


This colouring induces colourings $\chi_{i}$ on the copies of $\mathbf{E}_{i}$ in $\mathbf{C}_{2}$ for each $i$. By the above Ramsey construction, there is $\mathbf{B} \in\left(\begin{array}{c}\mathbf{C}_{2} \\ \mathbf{A}_{2}\end{array}\right)$ which is monochromatic with respect to each $\chi_{i}$. In other words, we have the following possibilities:

1. The ordering $\prec$ on $\mathbf{B}$ agrees with $<$ '.

2. The ordering $\prec$ on agrees with $<^{\prime}$ on edges and goes in the opposite direction on non-edges.

3. The ordering $\prec$ on $\mathbf{B}$ agrees with $<^{\prime}$ on non-edges and goes in the opposite direction on edges.

4. The ordering $\prec$ on $\mathbf{B}$ goes in the opposite direction.

Now, since $\mathbf{A}_{1}$ embeds in $\mathbf{B}$, the structure $(\mathbf{A},<)$ must embed in $\mathbf{B}$ with the order $<^{\prime}$ replaced by $\prec$ and the proof is complete.

Theorem 8.7. The age of $n * I_{\omega}^{*}$ satisfies the expansion property with respect to the age of $n * I_{\omega}$.

Proof. Let $\mathbf{A} \in \operatorname{Age}\left(n * I_{\omega}\right)$, fix a sequence of relations $P_{i}^{\mathbf{A}}$ corresponding to each part of $\mathbf{A}$, and fix first a convex linear ordering $<$ on $A$ that agrees with these relations.

For $1 \leqslant i \leqslant n$, define $\mathbf{E}^{i} \in \operatorname{Age}\left(n * I_{\omega}^{*}\right)$ to be the structure with universe $\{a, b\}$ with no edges and such that $a<b$ and $P_{i}^{\mathbf{E}^{i}}=\{a, b\}$.

Enumerate all convex orderings on $A$ that agree with $P_{i}^{\mathbf{A}}$ 's: $<_{1}, \ldots,<_{o}$. Find $\mathbf{B} \in$ $\operatorname{Age}\left(n * I_{\omega}^{*}\right)$ so that each $\left(\mathbf{A}, P_{1}^{\mathbf{A}}, \ldots, P_{n}^{\mathbf{A}},<_{i}\right)$ embeds in $\mathbf{B}$, for $1 \leqslant i \leqslant o$. Then find $\mathbf{D} \in \operatorname{Age}\left(n * I_{\omega}^{*}\right)$ so that $\mathbf{B}$ and all $E^{i}$ s embed in $\mathbf{D}$. Since $\operatorname{Age}\left(n * I_{\omega}^{*}\right)$ satisfies the Ramsey property, we can find $\mathbf{C}_{i} \in \operatorname{Age}\left(n * I_{\omega}^{*}\right)$ so that $\mathbf{C}_{1} \rightarrow(\mathbf{D})_{2}^{\mathbf{E}^{1}}, \mathbf{C}_{i+1} \rightarrow\left(\mathbf{C}_{i}\right)_{2}^{\mathbf{E}^{i+1}}$. Let $<^{\prime}$ be the ordering on $\mathbf{C}_{n}$, and let $\prec$ be any convex linear ordering on $C_{n}$ that agrees with $P_{i}^{\mathbf{C}_{n}}$ 's.

As before consider the following colourings on the copies of $\mathbf{E}^{i}$ in $\mathbf{C}_{n}$ :

$$
\chi_{i}\left(\mathbf{E}^{\prime}\right)= \begin{cases}0 & \text { if the two orders }<^{\prime} \text { and } \prec \text { agree on } E^{\prime} \\ 1 & \text { otherwise }\end{cases}
$$

By the Ramsey property we can thus find $\mathbf{D}^{\prime} \in\left(\begin{array}{c}\mathbf{C}_{n} \\ \mathbf{D}\end{array}\right)$ which is monochromatic with respect to $\chi_{1}, \ldots, \chi_{n}$. In other words, $\prec$ either agrees with $<^{\prime}$ or goes in the opposite direction when restricted to any part. Furthermore, $\prec$ already agrees with $<^{\prime}$ on edges, because we have assumed that $\prec$ 's convexity agrees with the relations $P_{i}^{\mathbf{C}_{n}}$. Now, since $\mathbf{B}$ embeds in $\mathbf{D}^{\prime}$, the structure $\left(\mathbf{A}, P_{1}^{\mathbf{A}}, \ldots, P_{n}^{\mathbf{A}},<\right)$ must embed in $\mathbf{D}^{\prime}$ with the order $<^{\prime}$ replaced by $\prec$.

Let $\mathbf{C}^{\prime}$ the reduct of $\mathbf{C}_{n}$ resulting from removing $<^{\prime}$. Given a bijection $f:[n] \rightarrow[n]$, define $\mathbf{C}_{f}$ to be $\mathbf{C}^{\prime}$ except that $P_{i}^{\mathbf{C}_{f}}=P_{f(i)}^{\mathbf{C}^{\prime}}$. Find a structure $\mathbf{C}^{\prime \prime}$ so that every $\mathbf{C}_{f}$ embeds into it. Set $\mathbf{C}_{0} \in \operatorname{Age}\left(n * I_{\omega}\right)$ to be the reduct of $\mathbf{C}^{\prime \prime}$. For any $P_{1}^{\mathbf{C}_{0}}, \ldots, P_{n}^{\mathbf{C}_{0}}$ and any convex linear order $<^{\prime \prime}$, we can find a convex linear order $<^{\prime \prime \prime}$ so that

$$
\left(\mathbf{C}^{\prime}, P_{1}^{\mathbf{C}^{\prime}}, \ldots, P_{n}^{\mathbf{C}^{\prime}},<^{\prime \prime \prime}\right)
$$


embeds in

$$
\left(\mathbf{C}_{0}, P_{1}^{\mathbf{C}_{0}}, \ldots, P_{n}^{\mathbf{C}_{0}},<^{\prime \prime}\right)
$$

by construction. Therefore,

$$
\left(\mathbf{A}, P_{1}^{\mathbf{A}}, \ldots, P_{n}^{\mathbf{A}},<\right)
$$

embeds in any expansion

$$
\left(\mathbf{C}_{0}, P_{1}^{\mathbf{C}_{0}}, \ldots, P_{n}^{\mathbf{C}_{0}},<^{\prime \prime}\right),
$$

given any $P_{1}^{\mathbf{C}_{0}}, \ldots, P_{n}^{\mathbf{C}_{0}}$, and any convex linear order $<"$.

Finally, to find the witness of the expansion property for $\mathbf{A}$, take the joint embedding of all $\mathbf{C}_{0}$ 's, for all sequences $P_{1}^{\mathbf{A}}, \ldots, P_{n}^{\mathbf{A}}$ and all convex linear orderings $<$ on $\mathbf{A}$.

\section{$9 \mathcal{P}(3)$}

Let $\mathcal{P}=(P, \prec)$ be the generic partial order. In this context, a subset $D$ of $\mathcal{P}$ is dense if and only if for every $x, z \in P$ there is $y \in D$ such that $x \prec y \prec z$ (see [C13]). Partition $P$ into three dense subsets $P_{0}, P_{1}, P_{2}$. Note that each $P_{i}$ is isomorphic as a partial order to $\mathcal{P}$. Define $\mathbb{P}$ to be the directed graph with vertex set $P$ so that $(x, y)$ is an edge if and only if $x \prec y$. Our underlying signature is $\mathcal{L}=\{E\}$ where $E$ is the binary directed edge relation. We will temporarily consider extra binary relations $T_{i}^{X}$, for $i \in\{-1,0,1\}$ and a set of pairs $X$. For a set of pairs $X$, define the relations $T_{i}^{X}$ as follows: put $(x, y) \in T_{-1}^{X}$ if and only if $(x, y) \in X$; put $(x, y) \in T_{1}^{X}$ if and only if $(y, x) \in X$; finally, put $(x, y) \in T_{0}^{X}$, otherwise. We define $\mathcal{P}(3)$ as a variant of $\mathbb{P}$ by "twisting" the directed edges $E^{\mathbb{P}}$ : The set of vertices of $\mathcal{P}(3)$ is $P$. Define the edges $E^{\mathcal{P}(3)}$ so that $(x, y) \in T_{k+(j-i) \bmod 3}^{E^{\mathcal{P}(3)}}$ if and only if $(x, y) \in T_{k}^{E^{\mathbb{P}}}$, whenever $x \in P_{i}$ and $y \in P_{j}$.

An important observation, which we will use to show the expansion property, is that a cycle of length three embeds in $\mathcal{P}(3)$, but not in $\mathcal{P}$.

Now, we define the expansion of $\mathcal{P}(3)$. Let $\mathcal{L}^{\prime}=\left\{E, R_{0}, R_{1}, R_{2},<\right\}$ be the signature, where $E$ and $<$ are binary and $R_{i}$ 's are unary. We set $E^{\mathcal{P}(3)^{*}}$ to be $E^{\mathcal{P}(3)}$. Define $\mathcal{P}(3)^{*}$ to be the expansion of $\mathcal{P}(3)$ where $R_{i}^{\mathcal{P}(3)^{*}}=P_{i}$ and $<^{\mathcal{P}(3)^{*}}$ is a linear extension of $\prec$ such that $\mathcal{P}(3)^{*}$ is still a Fraïssé structure. Define $\mathcal{K}$ to be the age of $\mathcal{P}(3)^{*}$.

Next, we define signatures

$$
\mathcal{L}_{0}=\left\{\prec, R_{0}, R_{1}, R_{2},<\right\}, \mathcal{L}_{1}=\{\prec,<\},
$$

and

$$
\mathcal{L}_{2}=\left\{R_{0}, R_{1}, R_{2},<\right\},
$$

where $R_{i}$ 's are unary, while $\prec$ and $<$ are binary.

Define $\mathcal{K}_{0}$ to be the class of finite structures $\left(A, \prec^{\mathbf{A}}, R_{0}^{\mathbf{A}}, R_{1}^{\mathbf{A}}, R_{2}^{\mathbf{A}},<^{\mathbf{A}}\right)$ where $\prec^{\mathbf{A}}$ is a poset on $A, R_{i}^{\mathbf{A}}$ 's partition $A$ and $<^{\mathbf{A}}$ is a linear order extending $\prec^{\mathbf{A}}$. Define $\mathcal{K}_{i}$ to be the class of reducts of structures from $\mathcal{K}_{0}$ with signature $\mathcal{L}_{i}$, for $i \in\{1,2\}$. The class $\mathcal{K}_{1}$ already satisfies the Ramsey property, see [PTW84]. Moreover, the class $\mathcal{K}_{2}$ is essentially 
the previously defined class $\mathcal{O P}_{3}$ and its Ramsey property was demonstrated in [KPT05] (see proof of Theorem 8.4).

The members of the class $\mathcal{K}_{0}$ can be treated as members of the class $\mathcal{K}$ and vice versa. For simplicity, we suppose a given $\mathbf{A} \in \mathcal{K}_{0}$ is such that $\left(A, \prec^{\mathbf{A}}\right)$ is a substructure of $\mathcal{P}$. By the "twisting" procedure defined at the beginning of this section, we arrive at a member $\mathbf{A}^{\prime}=\left(A, E^{\mathbf{A}^{\prime}}\right)$ of the age of $\mathcal{P}(3)$ so that $\left(A, E^{\mathbf{A}^{\prime}}, R_{0}^{\mathbf{A}}, R_{1}^{\mathbf{A}}, R_{2}^{\mathbf{A}},<^{\mathbf{A}}\right)$ is in the age of $\mathcal{P}(3)^{*}$. By reversing this procedure, we may turn a given member of $\mathcal{K}$ into a member of $\mathcal{K}_{0}$.

What follows is a short but powerful argument from [S13-2], which we include here to keep this paper as self-contained as possible, as before. The reader is invited to compare it to the proof of the result from [B12] found in [S12].

Theorem 9.1. The class $\mathcal{K}_{0}$ satisfies the Ramsey property.

Proof. Let

$$
\mathbf{A}=\left(A, E^{\mathbf{A}}, R_{0}^{\mathbf{A}}, R_{1}^{\mathbf{A}}, R_{2}^{\mathbf{A}},<^{\mathbf{A}}\right)
$$

and

be in $\mathcal{K}$. Set $\mathbf{A}_{1}=\left(A, E^{\mathbf{A}},<^{\mathbf{A}}\right)$,

$$
\mathbf{B}=\left(A, E^{\mathbf{B}}, R_{0}^{\mathbf{B}}, R_{1}^{\mathbf{B}}, R_{2}^{\mathbf{B}},<^{\mathbf{B}}\right)
$$

$$
\begin{gathered}
\mathbf{A}_{2}=\left(A, R_{0}^{\mathbf{A}}, R_{1}^{\mathbf{A}}, R_{2}^{\mathbf{A}},<^{\mathbf{A}}\right), \\
\quad \mathbf{B}_{1}=\left(A, E^{\mathbf{B}},<^{\mathbf{B}}\right), \\
\mathbf{B}_{2}=\left(A, R_{0}^{\mathbf{B}}, R_{1}^{\mathbf{B}}, R_{2}^{\mathbf{B}},<^{\mathbf{B}}\right) .
\end{gathered}
$$

Let $r$ be a natural number. We have $\mathbf{C}_{1} \in \mathcal{K}_{1}$ and $\mathbf{C}_{2} \in \mathcal{K}_{2}$ such that

$$
\left(\mathbf{C}_{1}, \mathbf{C}_{2}\right) \rightarrow\left(\mathbf{B}_{1}, \mathbf{B}_{2}\right)_{r}^{\left(\mathbf{A}_{1}, \mathbf{A}_{2}\right)}
$$

Define $\mathbf{C} \in \mathcal{K}$ as follows: Set the universe $C=C_{1} \times C_{2}$. Set $(x, y) \prec^{\mathbf{C}}(z, w)$ if and only if $x \prec^{\mathbf{C}_{1}} z$. Set $(x, y) \in R_{i}^{\mathbf{C}}$ if and only if $y \in R_{i}^{\mathbf{C}_{2}}$. Finally, set $(x, y)<^{\mathbf{C}}(z, w)$ if and only if $x<{ }^{\mathbf{C}_{1}} z$ or $\left(x=z\right.$ and $\left.y<{ }^{\mathrm{C}_{2}} w\right)$. For structures $\mathbf{D}_{1} \subseteq \mathbf{C}_{1}, \mathbf{D}_{2} \subseteq \mathbf{C}_{2}$ of the same size, define the diagonal substructure $\Delta\left(\mathbf{D}_{1}, \mathbf{D}_{2}\right) \subseteq \mathbf{C}$ as follows: First define an ordering on $\left[\left|D_{1}\right|\right] \times\left[\left|D_{2}\right|\right]$ by setting $(x, y)<(z, w)$ if and only if $x<z$ or $(x=z$ and $y<w)$. There is a unique order preserving map $f:\left[\left|D_{1}\right|\right] \times\left[\left|D_{2}\right|\right] \rightarrow D_{1} \times D_{2}$. Set the universe of $\Delta\left(\mathbf{D}_{1}, \mathbf{D}_{2}\right)$ to be $\left\{f(1,1), f(2,2), \ldots, f\left(\left|D_{1}\right|,\left|D_{2}\right|\right)\right\}$.

Let

$$
\chi:\left(\begin{array}{l}
\mathbf{C} \\
\mathbf{A}
\end{array}\right) \rightarrow[r] .
$$

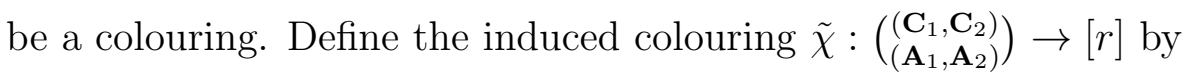

$$
\tilde{\chi}\left(\left(\mathbf{A}_{1}^{\prime}, \mathbf{A}_{2}^{\prime}\right)\right)=\chi\left(\Delta\left(\mathbf{A}_{1}^{\prime}, \mathbf{A}_{2}^{\prime}\right)\right) .
$$

Then there is a $\tilde{\chi}$-monochromatic

$$
\left(\mathbf{B}_{1}^{\prime}, \mathbf{B}_{2}^{\prime}\right) \in\left(\begin{array}{c}
\left(\mathbf{C}_{1}, \mathbf{C}_{2}\right) \\
\left(\mathbf{B}_{1}, \mathbf{B}_{2}\right)
\end{array}\right)
$$

Set $\mathbf{B}^{\prime}=\Delta\left(\mathbf{B}_{1}^{\prime}, \mathbf{B}_{2}^{\prime}\right)$. Then $\mathbf{B}^{\prime}$ is isomorphic to $\mathbf{B}$ and is $\chi$-monochromatic. 
Corollary 9.2. The age of $\mathcal{P}(3)^{*}$ satisfies the Ramsey property.

Theorem 9.3. The age of $\mathcal{P}(3)^{*}$ satisfies the expansion property with respect to the age of $\mathcal{P}(3)$.

Proof. We first define a list of elements of the age of $\mathcal{P}(3)^{*}$ :

- If $i \in\{0,1,2\}$, set $\mathbf{X}_{i}$ to be such that $X_{i}=\{x\}, E^{\mathbf{X}_{i}}=<^{\mathbf{X}_{i}}=\emptyset$. Moreover, set $R_{i}^{\mathbf{X}_{i}}=\{x\}$ and $R_{i}^{\mathbf{X}_{j}}=\emptyset$ for $i \neq j$.

- If $i \in\{0,1,2\}$, set $\mathbf{D}_{i}$ to be such that $D_{i}=\{x, y, z\}$,

$E^{\mathbf{D}_{i}}=\{(x, y),(y, z),(z, x)\}, R_{i}^{\mathbf{D}_{i}}=\{x, y\}, R_{(i+1) \bmod 3}^{\mathbf{D}_{i}}=\{z\}$,

$<^{\mathbf{D}_{i}}=\{(x, y),(y, z),(x, z)\}$.

- If $i, j \in\{0,1,2\}, i \leqslant j$, set $\mathbf{E}_{i, j}^{+}$to be such that $E_{i, j}^{+}=\{x, y\}, x \in R_{i}^{\mathbf{E}_{i, j}^{+}}, y \in R_{j}^{\mathbf{E}_{i, j}^{+}}$. Set $<^{\mathbf{E}_{i, j}^{+}}=\{(x, y)\}$. If $i=j$, set $E^{\mathbf{E}_{i, j}^{+}}=\emptyset$. If $j=i+1$, set $E^{\mathbf{E}_{i, j}^{+}}=\{(y, x)\}$. If $j=i+2$, set $E^{\mathbf{E}_{i, j}^{+}}=\{(x, y)\}$.

- If $i, j \in\{0,1,2\}, i<j$, set $\mathbf{E}_{i, j}^{-}$to be such that $E_{i, j}^{-}=\{x, y\}, x \in R_{i}^{\mathbf{E}_{i, j}^{-}}, y \in R_{j}^{\mathbf{E}_{i, j}^{-}}$. Set $<^{\mathbf{E}_{i, j}^{-}}=\{(y, x)\}$. If $j=i+1$, set $E^{\mathbf{E}_{i, j}^{-}}=\{(y, x)\}$. If $j=i+2$, set $E^{\mathbf{E}_{i, j}^{-}}=\{(x, y)\}$.

- For $i \in\{0,1,2\}$, set $\mathbf{F}_{i}$ to be such that $F_{i}=\{x, y, z\}$.

Set $<^{\mathbf{F}_{i}}=\{(x, y),(y, z),(x, z)\}$. Set $E^{\mathbf{F}_{i}}=\{(x, z)\}, R_{i}^{\mathbf{F}_{i}}=\{x, y, z\}, R_{k}^{\mathbf{F}_{i}}=\emptyset$ for $k \neq i$.

- For $i, j \in\{0,1,2\}, i \neq j$, set $\mathbf{G}_{i, j}$ to be such that $G_{i, j}=\{x, y, z\}, R_{i}^{\mathbf{G}_{i, j}}=$ $\{x, y\}, R_{j}^{\mathbf{G}_{i, j}}=\{z\},<\mathbf{G}_{i, j}=\{(z, x),(x, y),(z, y)\}$. If $j=i+1$, then set $E^{\mathbf{G}_{i, j}}=$ $\{(x, z),(y, z)\}$. If $j=i+2$, then set $E^{\mathbf{G}_{i, j}}=\{(x, y),(y, z)\}$. If $j=i-1$, then set $E^{\mathbf{G}_{i, j}}=\{(y, z)\}$. If $j=i-2$, then set $E^{\mathbf{G}_{i, j}}=\{(x, z),(z, y)\}$.

Note that $\mathbf{D}_{i}$ 's are cycles. Let $\mathbf{A}$ be in the age of $\mathcal{P}(3)^{*}$. Let $\mathbf{A}^{\prime}=\left(A, E^{\mathbf{A}}\right)$ be its reduct. List all possible expansions of $\mathbf{A}^{\prime}$ that result in elements of the age of $\mathcal{P}(3)^{*}: \mathbf{A}_{1}, \ldots \mathbf{A}_{k}$. Let $\mathbf{B}$ be a structure in the age of $\mathcal{P}(3)^{*}$ such that $\mathbf{A}, \mathbf{A}_{i}{ }^{\prime} \mathrm{s}, \mathbf{X}_{i}{ }^{\prime} \mathrm{s}, \mathbf{E}_{i, j}^{+/-}{ }^{\prime}$ s, $\mathbf{F}_{i}$ 's and $\mathbf{G}_{i, j}$ 's all embed in $\mathbf{B}$. We can find such a $\mathbf{B}$ due to the joint embedding property. Using the Ramsey property, we can find $\mathbf{C}_{i}$ in the age of $\mathcal{P}(3)^{*}$ such that

$$
\begin{gathered}
\mathbf{C}_{0} \rightarrow(\mathbf{B})_{3}^{\mathbf{X}_{0}} \\
\mathbf{C}_{1} \rightarrow\left(\mathbf{C}_{0}\right)_{3}^{\mathbf{X}_{1}} \\
\mathbf{C}_{2} \rightarrow\left(\mathbf{C}_{1}\right)_{3}^{\mathbf{X}_{2}} .
\end{gathered}
$$

Fix a bijection $g:\{0, \ldots, 5\} \rightarrow(\{0,1,2\} \times\{0,1,2\}) \backslash\{(i, j): i>j\}$. Find

$$
\mathbf{C}_{3+i} \rightarrow\left(\mathbf{C}_{2+i}\right)_{2}^{\mathbf{E}_{g(i)}^{+}}
$$

for $0 \leqslant i \leqslant 5$. 
Fix a bijection $h:\{0, \ldots, 2\} \rightarrow(\{0,1,2\} \times\{0,1,2\}) \backslash\{(i, j): i \geqslant j\}$. Find

$$
\mathbf{C}_{9+i} \rightarrow\left(\mathbf{C}_{8+i}\right)_{2}^{\mathbf{E}_{h(i)}^{-}}
$$

for $0 \leqslant i \leqslant 2$.

Set $\mathbf{C}$ to be $\mathbf{C}_{11}$. Let $\mathbf{C}^{\prime}=\left(C, E^{\mathbf{C}}\right)$ be a reduct of $\mathbf{C}$. Consider any expansion $\mathbf{C}^{*}$ of $\mathbf{C}^{\prime}$ in the age of $\mathcal{P}(3)^{*}$. For $i \in\{0,1\}$, define colourings $\chi_{i}:\left(\begin{array}{c}\mathbf{C} \\ \mathbf{X}_{i}\end{array}\right) \rightarrow\{0,1,2\}$ so that $\chi\left(\mathbf{X}^{\prime}\right)=i$ where $X^{\prime}=\{x\}$ and $x \in R_{i}^{\mathbf{C}^{*}}$. For $0 \leqslant i \leqslant 5$, define $\chi_{3+i}:\left(\begin{array}{c}\mathbf{C} \\ \mathbf{E}_{g(i)}^{+}\end{array}\right) \rightarrow\{0,1\}$ by setting $\chi_{3+i}\left(\mathbf{E}^{\prime}\right)=0$ whenever $\mathbf{E}^{\prime}=\{x, y\}, x<^{\mathbf{C}} y$ and $x<^{\mathbf{C}^{*}} y$. Also, set $\chi_{3+i}\left(\mathbf{E}^{\prime}\right)=1$ whenever $\mathbf{E}^{\prime}=\{x, y\}, x<<^{\mathbf{C}} y$ but $x>^{\mathbf{C}^{*}} y$. Likewise, for $0 \leqslant i \leqslant 3$, define $\chi_{9+i}:\left(\begin{array}{c}\mathbf{C} \\ \mathbf{E}_{g(i)}^{-}\end{array}\right) \rightarrow\{0,1\}$ by setting $\chi_{9+i}\left(\mathbf{E}^{\prime}\right)=0$ whenever $\mathbf{E}^{\prime}=\{x, y\}, x<^{\mathbf{C}} y$ and $x<\mathbf{C}^{*} y$. Furthermore, set $\chi_{9+i}\left(\mathbf{E}^{\prime}\right)=1$ whenever $\mathbf{E}^{\prime}=\{x, y\}, x<<^{\mathbf{C}} y$ but $x>^{\mathbf{C}^{*}} y$. We can find $\mathbf{B}^{\prime} \in\left(\begin{array}{l}\mathbf{C} \\ \mathbf{B}\end{array}\right)$ that is $\chi_{i}$-monochromatic, for all $i$. Set $k_{i}$ to be the colour assigned by $\chi_{i}$ for elements in $\left(\begin{array}{c}\mathbf{B}^{\prime} \\ \mathbf{x}_{i}\end{array}\right)$, for $i<3$.

We first show that the parts of $\mathbf{B}^{\prime}$ determined by $R_{i}$ 's remain the same in $\mathbf{C}^{*}$ (other than being perhaps reordered): more specifically, we show that $k_{i} \neq k_{j}$, for $i \neq j, i, j<3$. Suppose otherwise, i.e., $k_{i_{0}}=k_{j_{0}}$, for some $i_{0} \neq j_{0}, i_{0}, j_{0}<3$. Consider a cycle $\mathbf{D} \in\left(\begin{array}{c}\mathbf{B}^{\prime} \\ \mathbf{D}_{i_{0}}\end{array}\right)$. For all $x \in D$, we have $x \in R_{i_{0}}^{\mathbf{D}}$. This means that $\mathbf{D}$ embeds in $P_{i_{0}}$ which is impossible because $\left(P_{i_{0}}, \prec\right)$ is isomorphic to the generic partial order.

We can show that for every $i>3$, the colouring $\chi_{i}$ cannot assign the colour 1 on $\left(\begin{array}{c}\mathbf{B}^{\prime} \\ \mathbf{E}_{g(i)}^{+}\end{array}\right)$ or $\left(\begin{array}{c}\mathbf{B}^{\prime} \\ \mathbf{E}_{h(i)}^{-}\end{array}\right)$: Suppose otherwise. Let $k \in\{0,1,2\}$. Then $\mathbf{F}_{k}$ embeds in $\mathbf{B}^{\prime}$. If the colour of every copy of $\mathbf{E}_{k, k}^{+}$in $\mathbf{B}^{\prime}$ is assumed to be 1 , we have that $<^{\mathbf{C}^{*}}$ restricted to $F_{k} \times F_{k}$ is a cycle. This is absurd. This means that every "non-edge" in $\mathbf{B}^{\prime}$ entirely contained in a part has the same linear extension with respect to $\mathbf{C}^{*}$ and $\mathbf{C} .\left(^{*}\right)$

Let $k<\ell, k, \ell \in\{0,1,2\}$. Then $\mathbf{G}_{k, \ell}$ embeds in $\mathbf{B}^{\prime}$, as well. By $\left(^{*}\right)$, the single non-edge of every copy of $\mathbf{G}_{k, \ell}$ contained entirely in a part has only one possible linear extension. If the colour of every copy of $\mathbf{E}_{k, \ell}^{+}$in $\mathbf{B}^{\prime}$ is assumed to be 1 , we have that $<\mathbf{C}^{*}$ restricted to $G_{k, \ell} \times G_{k, \ell}$ would again be a cycle which is again absurd. The case when $k>\ell$ is similar, except we replace $\mathbf{E}_{k, \ell}^{+}$with $\mathbf{E}_{k, \ell}^{-}$.

In other words, the extension $<^{\mathbf{C}^{*}}$ agrees with $<^{\mathbf{C}}$ when both are restricted to $\mathbf{B}^{\prime}$. Thus, by construction of $\mathbf{B}$ and since $\mathbf{B} \cong \mathbf{B}^{\prime}$, any expansion of $\mathbf{A}^{*}$ embeds in $\mathbf{B}^{\prime}$ and therefore in $\mathbf{C}^{*}$.

\section{Semigeneric}

The semigeneric directed graph is the Fraïssé limit of the class $\mathcal{S}$ made of all those elements of Age $\left(\omega * I_{\omega}\right)$ which satisfy the following parity constraint: for any two distinct parts $P$ and $P^{\prime}$, distinct $u, v$ in $P$ and distinct $x, y$ in $P^{\prime}$, the number of edges directed from $u, v$ to $x, y$ is even.

We construct an expansion $\mathcal{S}^{*}$ of $\mathcal{S}$ by adding two binary relations symbols, $R$ and $<$. As usual, the symbol $<$ will be interpreted as a linear order. Let $\mathbf{A} \in \mathcal{S}$ be $k$-partite. 
Before explaining how an expansion of $\mathbf{A}$ is built, let us point out a crucial fact: given two different parts $P$ and $P^{\prime}$ of $\mathbf{A}$, parity constraints imply that the following relation is well-defined, and is an equivalence relation on $P$ :

$$
u \sim_{P^{\prime}}^{\mathbf{A}} v \quad \text { iff } \quad \forall x \in P^{\prime} E^{\mathbf{A}}(x, u) \leftrightarrow E^{\mathbf{A}}(x, v)
$$

In fact there are two equivalence classes, one is $\left\{u \in P: E^{\mathbf{A}}(x, u)\right\}$ and the other is $\left\{v \in P: E^{\mathbf{A}}(v, x)\right\}$, and observe these two classes do not depend on the choice of $x \in P^{\prime}$. Moreover the relation $\sim^{\mathbf{A}}$ defined on $A$ by:

$$
u \sim^{\mathbf{A}} v \quad \text { iff } \quad \exists P \quad\{u, v\} \subseteq P \quad \text { and } \quad \forall P^{\prime} \neq P u \sim_{P^{\prime}}^{\mathbf{A}} v
$$

is also an equivalence relation. Note that the number of $\sim^{\mathbf{A}}$-classes is at most $k 2^{k-1}$ (at most $2^{k-1}$ classes on each part), regardless of the actual size of $\mathbf{A}$, but will eventually reach this maximum size by the joint embedding property.

We now explain how an expansion $\mathbf{A}^{*}$ is constructed. We first consider a linear order $T=\left\{t_{i}: i<k\right\}$, viewed as a directed graph, where there is an edge from $t_{i}$ to $t_{j}$ whenever $t_{i}<t_{j}$. This is an element of $\mathcal{S}$, and thus by the joint embedding property we can take a $k$-partite element $\overline{\mathbf{A}}$ of $\mathcal{S}$ whose universe is the disjoint union of $A$ and $T$ and into which both $\mathbf{A}$ and $\mathbf{T}$ embed, and thus $T$ becomes a transversal in $\overline{\mathbf{A}}$. Next, for $u$ and $x$ in different parts of $\mathbf{A}$, we define

$$
R^{\mathbf{A}^{*}}(x, u) \quad \text { iff } \quad E^{\overline{\mathbf{A}}}\left(t_{i}, u\right)
$$

where $t_{i}$ is the only element of $T$ lying in the same part of $\overline{\mathbf{A}}$ as $x$. Finally, consider a linear order on $\overline{\mathbf{A}}$ that extends the already existing linear order on the copy of $T$ and is convex relative to the $k$-partition of $\overline{\mathbf{A}}$ (but not necessarily with respect to $\sim^{\overline{\mathbf{A}}}$, which is finer). Then finally let $<^{\mathbf{A}^{*}}$ denote its restriction on $\mathbf{A}$.

Note that adding $R^{\mathbf{A}^{*}}$ and $<^{\mathbf{A}^{*}}$ as above corresponds essentially to adding unary predicates $P_{i, f}^{\mathbf{A}^{*}}$, with $i<k$ and $f \in[2]^{[k] \backslash\{i\}}$, that partition $\mathbf{A}$ into the $\sim^{\mathbf{A}}$ equivalence classes. Indeed, $<\mathbf{A}^{*}$ labels the parts of $\mathbf{A}$ as $P_{0}<\mathbf{A}^{*} \cdots<\mathbf{A}^{*} P_{k-1}$, and $R^{\mathbf{A}^{*}}$ gives rise to:

$$
P_{i, f}^{\mathbf{A}^{*}}=\left\{u \in P_{i}: \forall j \neq i\left(f(j)=0 \leftrightarrow \forall x \in P_{j} R^{\mathbf{A}^{*}}(x, u)\right)\right\} .
$$

Note also that the edge relation of $\overline{\mathbf{A}}$ can be recovered from $R^{\mathbf{A}^{*}}$ and $<^{\mathbf{A}^{*}}$, and hence from $\left(P_{i, f}^{\mathbf{A}^{*}}\right)_{i, f}$ and $<^{\mathbf{A}^{*}}$ as well. To see this, let $x, u \in \bar{A}$ be in different parts. If both of them belong to the transversal that was used to define $\overline{\mathbf{A}}$, then the edge relation is given by the order relation $<\mathbf{A}^{*}$ between any two elements of $\mathbf{A}^{*}$ from the respective parts. If exactly one of them belongs to the transversal set, say $x=t_{i}$, then knowing whether there is an edge from $x=t_{i}$ to $u$ is directly available from the relation $R^{\mathbf{A}^{*}}$ evaluated at $(y, u)$ for any $y \in A$ in the same part as $x=t_{i}$. On the other hand, if neither $x$ nor $u$ are in the transversal set, then consider the quadruple formed by $x, u$, and the corresponding elements $t_{x}$ and $t_{u}$ from the transversal set; in that quadruple, all edges but the one between $x$ and $u$ are available from $R^{\mathbf{A}^{*}}$ and $<^{\mathbf{A}^{*}}$ as described above, and so the orientation of the edge between $x$ and $u$ can be deduced thanks to the parity constraint. 
One last comment: any element of the class of $k$-sequences of members of $\mathcal{O P}{2^{k-1}}_{\text {whose }}$ unary predicates are indexed by the set of all pairs $(i, f)$ with $i<k$ and $f \in[2]^{[k] \backslash\{i\}}$ gives raise to a $k$-partite element of $\mathcal{S}^{*}$. Let us illustrate this on an example that will also be useful later on. Consider the set $S[n, k]$ defined as the collection of triples $(i, f, j)$ where $i<k, f \in[2]^{[k] \backslash\{i\}}$ and $j<n$. For $f$ and $i$ fixed, define

$$
P_{i, f}^{\mathbf{S}[n, k]^{*}}=\{(i, f, j): j<n\} .
$$

The element of $\mathcal{S}^{*}$ we are about to construct will be $k$-partite with parts:

$$
P_{i}^{\mathbf{S}[n, k]^{*}}=\bigcup_{f \in[2]]^{k] \backslash\{i\}}} P_{i, f}^{\mathbf{S}[n, k]^{*}}=\left\{(i, f, j): f \in[2]^{[k] \backslash\{i\}}, j<n\right\} .
$$

If, for each $i$, we equip $[2]^{[k] \backslash\{i\}}$ with the lexicographical order, then we can induce on $S[n, k]$ a natural lexicographical order $<^{\mathbf{S}[n, k]^{*}}$. This is clearly convex.

To define $R^{\mathbf{S}[n, k]^{*}}$, set:

$$
R^{\mathbf{S}[n, k]^{*}}\left(\left(i^{\prime}, f^{\prime}, j^{\prime}\right),(i, f, j)\right) \quad \text { iff } \quad i \neq i^{\prime} \text { and } f\left(i^{\prime}\right)=0 .
$$

For the edge relation $E^{\mathbf{S}[n, k]^{*}}$, we introduce an imaginary transversal $\left\{t_{i}: i<k\right\}$, where each imaginary point $t_{i^{\prime}}$ is attached to the part $P_{i^{\prime}}^{\mathbf{S}[n, k]^{*}}$. First, add an edge from $t_{i^{\prime}}$ to $t_{i}$ whenever $i^{\prime}<i$. Then for each $(i, f, j)$ with $i \neq i^{\prime}$, add an edge from $t_{i^{\prime}}$ to $(i, f, j)$ iff $f\left(i^{\prime}\right)=0$, i.e. whenever $R^{\mathbf{S}[n, k]^{*}}\left(\left(i^{\prime}, f^{\prime}, j^{\prime}\right),(i, f, j)\right)$ for every point of the form $\left(i^{\prime}, f^{\prime}, j^{\prime}\right)$. Otherwise, add an edge in the other direction. Next, when $(i, f, j)$ and $\left(i^{\prime}, f^{\prime}, j^{\prime}\right)$ are such that $i \neq i^{\prime}$, consider the quadruple formed by $(i, f, j),\left(i^{\prime}, f^{\prime}, j^{\prime}\right), t_{i}$ and $t_{i^{\prime}}$. There is only one way to add an oriented edge between $(i, f, j)$ and $\left(i^{\prime}, f^{\prime}, j^{\prime}\right)$ in such a way that the number of edges from the pair $\left\{(i, f, j), t_{i}\right\}$ to the pair $\left\{\left(i^{\prime}, f^{\prime}, j^{\prime}\right), t_{i^{\prime}}\right\}$ is even.

This is enough to ensure that the resulting directed graph structure $E^{\mathbf{S}[n, k]^{*}}$ is in $\mathcal{S}$ : indeed, consider a quadruple formed by pairs $\{u, v\},\{x, y\}$ of elements in same parts. Add the corresponding imaginary points $t$ and $t^{\prime}$. For each transversal pair of $\{u, v, x, y\}$, complete it into a quadruple using $\left\{t, t^{\prime}\right\}$ where the parity constraint is satisfied. Identifying $E^{\mathbf{S}[n, k]^{*}}$ with its characteristic function, this is equivalent to saying some sum equals 0 modulo 2 . For example, for $\{u, x\}$ :

$$
E^{\mathbf{S}[n, k]^{*}}(u, x)+E^{\mathbf{S}[n, k]^{*}}\left(u, t^{\prime}\right)+E^{\mathbf{S}[n, k]^{*}}(t, x)+E^{\mathbf{S}[n, k]^{*}}\left(t, t^{\prime}\right)=0 .
$$

Adding up all these equalities for all transversal pairs of $\{u, v, x, y\}$ (i.e. pairs with elements in different parts), notice that each term involving $t$ or $t^{\prime}$ appears an even number of times, and therefore vanishes in the total sum. As a result, we obtain:

$$
E^{\mathbf{S}[n, k]^{*}}(u, x)+E^{\mathbf{S}[n, k]^{*}}(u, y)+E^{\mathbf{S}[n, k]^{*}}(v, x)+E^{\mathbf{S}[n, k]^{*}}(v, y)=0 .
$$

This shows that the parity constraint is satisfied on $\{u, v, x, y\}$, and shows that $\mathbf{S}[n, k]^{*} \in$ $\mathcal{S}^{*}$. We call $\mathbf{S}[n, k]$ the corresponding reduct in $\mathcal{S}$. Let us emphasize the following property of $\mathbf{S}[n, k]$ : 
Lemma 10.1. Every part of $\boldsymbol{S}[n, k]$ is partitioned into $2^{k-1}$ many $\sim^{S[n, k]}$-classes, each of size $n$.

Proof. Fix $i<k$. By construction, the $\sim^{\mathbf{S}[n, k]}$-classes inside the $i$-th part of $\mathbf{S}[n, k]$ are the sets $P_{i, f}^{\mathbf{S}[n, k]^{*}}=\{(i, f, j): j<n\}$, where $f$ runs over $[2]^{[k] \backslash\{i\}}$. Clearly, each has size $n$.

We define $\mathcal{A} S$ to consist of the elements of $\mathcal{S}^{*}$ with the ordering removed. Remembering how an expansion in $\mathcal{S}^{*}$ is intuitively built (by adding a transversal), a direct consequence of the previous lemma is the following corollary:

Corollary 10.2. All expansions of $\boldsymbol{S}[n, k]$ in $\mathcal{A} S$ are isomorphic.

We will use this result later on to prove the expansion property of $\mathcal{S}^{*}$ relative to $\mathcal{S}$. Before that, here is another direct consequence of the various facts we stated above, together with the Ramsey property for $k$-sequences of members $\mathcal{O P}_{2^{k-1}}$ (see Lemma 3.3):

Corollary 10.3. Suppose $\mathbf{A}^{*}, \mathbf{B}^{*} \in \mathcal{S}^{*}$ are $k$-partite. Then for any $r$ there exists a $k$ partite $\mathbf{C}^{*} \in \mathcal{S}^{*}$ such that,

$$
\mathbf{C}^{*} \rightarrow\left(\mathbf{B}^{*}\right)_{r}^{\mathbf{A}^{*}}
$$

With this fact in mind, we now prove that $\mathcal{S}^{*}$ has the Ramsey property.

Corollary 10.4. Suppose $\mathbf{A}^{*}, \mathbf{B}^{*} \in \mathcal{S}^{*}$ are $k$-partite and $\ell$-partite respectively. Then for any $r$ there exists an $\ell$-partite $\mathbf{C}^{*} \in \mathcal{S}^{*}$ such that any $r$-colouring $\chi$ of the copies of $\mathbf{A}^{*}$ with a fixed trace $\tau$, there exists a $\chi$-monochromatic $\tilde{\mathbf{B}} \in\left(\begin{array}{l}\mathbf{C}^{*} \\ \mathbf{B}^{*}\end{array}\right)$.

We symbolize this by

$$
\mathbf{C}^{*} \rightarrow\left(\mathbf{B}^{*}\right)_{r}^{\mathbf{A}^{*}, \tau} .
$$

Proof. Let $\mathbf{D}^{*}$ be the graph induced by the the elements of $\mathbf{B}^{*}$ with trace $\tau$. Use Corollary 10.3 , to get a $k$-partite $\mathbf{E}^{*}$ such that

$$
\mathbf{E}^{*} \rightarrow\left(\mathbf{D}^{*}\right)_{r}^{\mathbf{A}^{*}}
$$

Now extend each copy of $\mathbf{D}^{*}$ in $\mathbf{E}^{*}$ to a copy of $\mathbf{B}^{*}$ with trace $[\ell]$, via amalgamation. Finally, add edges outside of these copies, as necessary.

Corollary 10.5. Suppose $\mathbf{A}^{*}, \mathbf{B}^{*} \in \mathcal{S}^{*}$ are $k$-partite and $\ell$-partite respectively. Then for any $r$ there exists an $\ell$-partite $\mathbf{C}^{*} \in \mathcal{S}^{*}$ such that for any $r$-colouring $\chi$ of the copies of $\mathbf{A}^{*}$, there exists $\tilde{\mathbf{B}} \in\left(\begin{array}{l}\mathbf{C}^{*} \\ \mathbf{B}^{*}\end{array}\right)$ such that the colouring $\chi$ restricted to $\left(\begin{array}{c}\tilde{\mathbf{B}} \\ \mathbf{A}^{*}\end{array}\right)$ depends only on the trace. We symbolize this as

$$
\mathbf{C}^{*} \rightarrow\left(\mathbf{B}^{*}\right)_{r}^{\mathbf{A}^{*}, \operatorname{tr}}
$$

Proof. Enumerate subsets of $[\ell]$ of size $k: \tau_{1}, \ldots, \tau_{n}$. Set $\mathbf{C}_{1}^{*} \rightarrow\left(\mathbf{B}^{*}\right)_{r}^{\mathbf{A}^{*}, \tau_{1}}$. Set $\mathbf{C}_{i}^{*} \rightarrow$ $\left(\mathbf{C}_{i-1}^{*}\right)^{\mathbf{A}^{*}, \tau_{i}}$ for $i>1$. Finally, set $\mathbf{C}^{*}=\mathbf{C}_{n}^{*}$.

We are now ready for the full Ramsey result for $\mathcal{S}^{*}$. 
Theorem 10.6. The age of $\mathcal{S}^{*}$ is Ramsey.

Proof. Suppose $\mathbf{A}^{*}, \mathbf{B}^{*} \in \mathcal{S}^{*}$, and $r$ are given. We show that there exists an $\tilde{C}^{*} \in \mathcal{S}^{*}$ such that

$$
\mathbf{C}^{*} \rightarrow\left(\mathbf{B}^{*}\right)_{r}^{\mathbf{A}^{*}}
$$

First we apply Ramsey's theorem to the traces and use Corollary 10.5. Let $k \leqslant \ell \in \mathbb{N}$ such that $\mathbf{B}^{*}$ is $\ell$-partite and $\mathbf{A}^{*}$ is a $k$-partite substructure of $\mathbf{B}^{*}$. Using Ramsey's theorem, we can find $m$ such that $m \rightarrow(\ell)_{r}^{k}$. Enumerate the $\ell$-subsets of $[m]$ as $\tau_{1}, \ldots, \tau_{\ell}$. For each $i \leqslant \ell$ consider a copy $\mathbf{B}_{i}^{*}$ of $\mathbf{B}$ with trace $\tau_{i}$ in the Fraïssé limit $\mathbf{S}^{*}$ of $\mathcal{S}^{*}$ so that the $\mathbf{B}_{i}^{*}$ s are pairwise disjoint. Set $\overline{\mathbf{B}}^{*}$ to be the $m$-partite substructure of $\mathbf{S}^{*}$ supported by the union of these $\mathbf{B}_{i}^{*}$ s.

Using corollary 10.5 , we can find an $m$-partite $\mathbf{C}^{*}$ in $\mathbf{S}^{*}$ such that every $r$-colouring $\chi$ of $\left(\begin{array}{c}\mathbf{C}^{*} \\ \mathbf{A}^{*}\end{array}\right)$, there exists a copy $\overline{\mathbf{B}}^{\prime} \in\left(\begin{array}{c}\mathbf{C} \\ \overline{\mathbf{B}}^{*}\end{array}\right)$ such that $\chi$ restricted to $\left(\begin{array}{c}\overline{\mathbf{B}}^{\prime} \\ \mathbf{A}^{*}\end{array}\right)$ depends only on the trace.

Define $\bar{\chi}:\left(\begin{array}{c}m \\ k\end{array}\right) \rightarrow[r]$ by setting $\bar{\chi}(\sigma)$ to be the colour of any copy of $\mathbf{A}^{*}$ in $\overline{\mathbf{B}}^{\prime}$ with trace $\sigma$. This is well defined by construction. By choice of $m$, there is a $\bar{\chi}$-homogeneous $\tau_{i_{0}}$ of size $\ell$. By construction, there is $\mathbf{B}^{\prime} \in\left(\begin{array}{l}\overline{\mathbf{B}}^{\prime} \\ \mathbf{B}^{*}\end{array}\right)$ with trace $\tau_{i_{0}}$, which completes the proof.

It remains to show that $\mathcal{S}^{*}$ has the expansion property relative to $\mathcal{S}$. In order to do this, fix $\mathbf{A} \in \mathcal{S}$ as well as an expansion $\mathbf{A}^{*}$ of $\mathbf{A}$ in $\mathcal{S}^{*}$. We will be done once the following two lemmas are proved:

Lemma 10.7. There exists $\overline{\boldsymbol{B}} \in \mathcal{A S}$ for which any expansion of $\overline{\boldsymbol{B}}$ in $\mathcal{S}^{*}$ contains $\boldsymbol{A}^{*}$.

Proof. Consider all the expansions $\mathbf{A}_{0}^{*}, \ldots, \mathbf{A}_{s}^{*}$ of $\mathbf{A}$ obtained from $\mathbf{A}^{*}$ by reversing $<\mathbf{A}^{*}$ on some parts. Let $\hat{\mathbf{A}}^{*}$ be $k$-partite and containing them all. Then $\mathbf{A}^{*}$ embeds in $\hat{\mathbf{A}}^{*}$, even when $<\hat{\mathbf{A}}^{*}$ is reversed in any of its parts. Next, use the Ramsey property in $\mathcal{S}^{*}$ with colourings of edges and non-edges as in Theorem 8.6 to create $\overline{\mathbf{B}}^{*}$ so that any convex linear ordering $\prec$ on $\overline{\mathbf{B}}^{*}$ is so that $\left(\overline{\mathbf{B}}^{*}, E^{\overline{\mathbf{B}}^{*}}, R^{\overline{\mathbf{B}}^{*}}, \prec\right)$ contains a copy of $\hat{\mathbf{A}}^{*}$ up to a reversing of $\prec$ in some of the parts. Then, the reduct $\overline{\mathbf{B}}$ of $\overline{\mathbf{B}}^{*}$ in $\mathcal{A S}$ is as required.

Lemma 10.8. There exists $\boldsymbol{B} \in \mathcal{S}$ for which any expansion in $\mathcal{A S}$ contains $\overline{\boldsymbol{B}}$.

Proof. Observe that for $k$ and $n$ large enough, we can embed the reduct of $\overline{\mathbf{B}}$ in $\mathbf{S}[n, k]$. Then $\mathbf{S}[n, k]$ has the required property thanks to Corollary 10.2.

\section{Conclusion}

Having constructed Ramsey precompact expansions of all homogeneous directed graphs and having verified the expansion property in each case, we are automatically given a list of the respective universal minimal flows. Suppose $\mathbf{F}$ is one of the homogeneous directed graphs and $\mathbf{F}^{*}=\left(\mathbf{F}, \overrightarrow{R^{*}}\right)$ is its precompact expansion which we have constructed. The age of $\mathbf{F}^{*}$ satisfies the Ramsey property as well as the respective expansion property and 
consists of rigid elements. Consequently, its automorphism group is extremely amenable. Let $G$ be the automorphism group of $\mathbf{F}$. Moreover, recall that the logic action of $\mathbf{G}$ on $\overrightarrow{\mathrm{G} \cdot \overrightarrow{R^{*}}}$ is the universal minimal flow (see Theorem 1.2). In particular, we arrive at the following table, summarizing the results of this paper, where we omit the cases when $\mathbf{T}$ is finite (in these cases refer to the the definitions preceding Theorem 5.7 and Theorem 7.8).

\begin{tabular}{|c|c|}
\hline Homogeneous directed graph(s) F & Expanded language $\overrightarrow{R^{*}}$ \\
\hline $\mathbf{T}\left[I_{n}\right], I_{n}[\mathbf{T}] \hat{\mathbf{T}}$ & $\begin{array}{l}\text { Relations giving the expanded tour- } \\
\text { nament } \mathbf{T}^{*} \text { the Ramsey property and } \\
\text { the expansion property; } R_{1}^{\mathbf{F}^{*}}, \ldots, R_{n}^{\mathbf{F}^{*}} \\
\text { (unary relations which are transversals } \\
\text { in the case of } \mathbf{T}\left[I_{n}\right] \text {, distinguishing copies } \\
\text { of } \mathbf{T} \text { in the case of } I_{n}[\mathbf{T}] \text {, or the copies } \\
\text { of the generic partial order in the case } \\
\text { of } \mathcal{P}(3) \text { ), and }<^{*} \text { (convex linear ordering } \\
\text { on } \mathbf{F}^{*} \text { ) }\end{array}$ \\
\hline $\mathcal{P}(3)$ & $\begin{array}{l}R_{0}^{\mathbf{F}^{*}}, R_{1}^{\mathbf{F}^{*}}, R_{2}^{\mathbf{F}^{*}} \text { (unary relations distin- } \\
\text { guishing the copies of the generic partial } \\
\text { order), and }<^{*} \text { (linear ordering extend- } \\
\text { ing the underlying generic partial order) }\end{array}$ \\
\hline $\mathbf{T}\left[I_{\omega}\right], I_{\omega}[\mathbf{T}]$ & $\begin{array}{l}\text { Relations giving the expanded tourna- } \\
\text { ment } \mathbf{T}^{*} \text { the Ramsey property and the } \\
\text { expansion property; }<^{*} \text { (convex linear } \\
\text { ordering on } \mathbf{F}^{*} \text { ) }\end{array}$ \\
\hline$n * I_{\omega}$ & $\begin{array}{l}P_{1}^{\mathbf{F}^{*}}, \ldots, P_{n}^{\mathbf{F}^{*}} \text { (unary relations distin- } \\
\text { guishing each part of } n * I_{n} \text { ), and }<^{*} \\
\text { (convex linear ordering on } \mathbf{F}^{*} \text { ) }\end{array}$ \\
\hline$\omega * I_{\omega}$ & $<^{*}\left(\right.$ convex linear ordering on $\left.\mathbf{F}^{*}\right)$ \\
\hline semigeneric & $\begin{array}{l}<^{*}\left(\text { convex linear ordering on } \mathbf{F}^{*}\right), \text { and } \\
\left.R^{\mathbf{F}^{*}} \text { (sub-partitioning binary relation }\right)\end{array}$ \\
\hline
\end{tabular}

Note that the relations including the linear orders in the above table arise from taking the Fraïssé limits of our expanded classes. Note that the semigeneric case is the only one where a special binary relation was used (in addition to a linear order).

Completing the list of Ramsey precompact expansions of homogeneous structures and subsequently computing their universal minimal flows is an ever continuing undertaking. For example, we have examples of homogeneous metric spaces whose Ramsey precompact expansions are still unknown. In particular from the work of Cherlin in [C13], and related more closely to the subject of this paper, are classes of metrically homogeneous graphs, i.e., ones that are homogeneous with respect to the graph metric. 


\section{Acknowledgements}

This project was finished while both of us, Claude Laflamme and Lionel Nguyen Van Thé, were attending the thematic program Universality and Homogeneity at the Hausdorff Research Institute for Mathematics in Bonn. We would therefore like to acknowledge the support of the Hausdorff Research Institute for Mathematics, and thank the organizers Alexander Kechris, Katrin Tent and Anatoly Vershik for having made this stay possible. We would also like to thank Miodrag Sokić for his numerous suggestions.

\section{References}

[AH78] F. G. Abramson and L. A. Harrington, Models without indiscernibles. J. Symbolic Logic 43 (1978), no. 3, 572-600.

[B12] M. Bodirsky, New Ramsey classes from old. Electron. J. Combin. 21 (2014), no. 2. \#P2.22.

[C98] G. L. Cherlin, The classification of countable homogeneous directed graphs and countable homogeneous n-tournaments. Mem. Amer. Math. Soc. 131 (1998), no. 621.

[C13] G. L. Cherlin, Metrically Homogeneous Graphs, in preparation, 2013.

[F97] W. L. Fouché, Symmetry and the Ramsey degree of posets, Discrete Math. 167/168 (1997), 309-315.

[GRS90] R. Graham, B. Rothschild, J. Spencer. Ramsey Theory. 2nd ed. Wiley, New York (1990).

[KPT05] A. S. Kechris, V. G. Pestov, and S. Todorčević, Fraïssé limits, Ramsey theory, and topological dynamics of automorphism groups. Geom. Funct. Anal. 15 (2005), no. 1, 106-189.

[LNS10] C. Laflamme, L. Nguyen Van Thé and N. W. Sauer, Partition properties of the dense local order and a colored version of Milliken's theorem. Combinatorica 30 (2010), no. 1, 83-104.

[LW80] A. H. Lachlan and R. E. Woodrow, Countable ultrahomogeneous undirected graphs. Trans. Amer. Math. Soc. 262 (1980), no. 1, 51-94.

[N96] J. Nešetřil, Ramsey theory. Handbook of Combinatorics (vol. 2) (1996), 13311403.

[NR89] J. Nešetřil and V. Rödl, The partite construction and Ramsey set systems. Discrete Mathematics 75(1-3) (1989), 327-334.

[NR77] J. Nešetřil and V. Rödl, Partitions of finite relational and set systems. J. Combinatorial Theory Ser. A 22 (1977), no. 3, 289-312.

[NR83] J. Nešetřil and V. Rödl, Ramsey classes of set systems. J. Combin. Theory Ser. A 34 (1983), no. 2, 183-201. 
[NVT13] L. Nguyen Van Thé, More on the Kechris-Pestov-Todorcevic correspondence: precompact expansions. Fund. Math. 222 (2013), 19-47.

[PTW84] M. Paoli, W. T. Trotter Jr. and J. W. Walker, Graphs and orders in Ramsey theory and in dimension theory. Graphs and order (Banff, Alta., 1984), NATO Adv. Sci. Inst. Ser. C Math. Phys. Sci., vol 147, Reidel, Dordrecht, 1985, 351394.

[S12] M. Sokić, Ramsey Properties of Finite Posets II. Order 29 (2012), no. 1, 31-47.

[S13-1] M. Sokić, Directed graphs and boron trees, preprint.

[S13-2] M. Sokić, Ramsey degrees, labeled and unlabeled partitions, preprint.

[S13-3] M. Sokić, Relational quotients. Fundamenta Mathematicae, 221 (3). (2013) pp. 189-220. 